\title{
The JAK2-STAT3 pathway controls a beneficial proteostasis response of reactive astrocytes in Huntington's disease
}

\author{
Abjean L. ${ }^{1}$, Ben Haim L. ${ }^{1 *}$, Riquelme-Perez M. ${ }^{1,2^{*}}$, Gipchtein P. ${ }^{1}$, Derbois C. ${ }^{2}$, Palomares M.A. ${ }^{2}$, \\ Petit F. ${ }^{1}$, Hérard A.S. ${ }^{1}$, Gaillard M.C. ${ }^{1}$, Guillermier M. ${ }^{1}$, Gaudin-Guérif M. ${ }^{1}$, Sagar N. ${ }^{1}$, Dufour N. ${ }^{1}$, \\ Robil N. ${ }^{3}$, Kabani M. ${ }^{1}$, Melki R. ${ }^{1}$, De la Grange P. ${ }^{3}$, Bemelmans A.P. ${ }^{1}$, Bonvento G. ${ }^{1}$, Deleuze J.F. ${ }^{2}$, \\ Hantraye P. ${ }^{1}$, Bonnet E. ${ }^{2}$, Brohard S. ${ }^{2}$, Olaso R. ${ }^{2}$, Brouillet E. ${ }^{1}$, Carrillo-de Sauvage M.A. ${ }^{1}$, \\ Escartin C. ${ }^{1}$

\footnotetext{
${ }^{1}$ Université Paris-Saclay, Commissariat à l'Energie Atomique et aux Energies Alternatives, Centre National de la Recherche Scientifique, MIRCen, Laboratoire des Maladies Neurodégénératives, 92265, Fontenay-aux-Roses, France.

${ }^{2}$ Université Paris-Saclay, Commissariat à l'Energie Atomique et aux Energies Alternatives, Centre National de Recherche en Génomique Humaine, 91057, Evry, France.

${ }^{3}$ GenoSplice technology, 75013 Paris, France
}

*Equal contribution

\section{Short title}

STAT3-mediated proteostasis in HD astrocytes

Key words

Huntington's disease/ JAK2-STAT3 pathway/ Proteostasis/ Reactive astrocytes/ Viral vectors

\section{Corresponding author}

Carole Escartin, Ph.D.

UMR9199 - MIRCen

CNRS - CEA - Université Paris Saclay

18 , route du Panorama

92260 Fontenay-aux-roses

carole.escartin@cea.fr

Competitive interests: The authors declare no competing interests. 


\section{Abstract}

2

3 Huntington's disease (HD) is a fatal neurodegenerative disease characterized by striatal 4 neurodegeneration, aggregation of mutant Huntingtin (mHTT) and the presence of reactive astrocytes.

5 Astrocytes are important partners for neurons and engage in a specific reactive response in HD that

6 involves morphological, molecular and functional changes. How reactive astrocytes contribute to HD is

$7 \quad$ still an open question, especially because their reactive state is poorly reproduced in mouse models.

8 Here, we show that the JAK2-STAT3 pathway, a central cascade controlling the reactive response 9 of astrocytes, is activated in the putamen of HD patients. Selective activation of this cascade in astrocytes reduces the number and size of neuronal mHTT aggregates and improves neuronal features in two HD mouse models. Moreover, activation of the JAK2-STAT3 pathway in astrocytes coordinates a 12 transcriptional program that increases their intrinsic proteolytic capacity, through the lysosomes and the 13 ubiquitin-proteasome system, and enhances their production of the co-chaperone DNAJB1, which is 14 released in exosomes.

Together, our results show that the JAK2-STAT3 pathway controls a beneficial proteostasis response in reactive astrocytes in $\mathrm{HD}$, which involves bi-directional signalling with neurons to reduce mHTT aggregation and toxicity. 


\section{Introduction}

Huntington's disease (HD) is a genetic neurodegenerative disease that causes involuntary movements, psychiatric symptoms and cognitive deficits, with no curative treatment yet available ${ }^{1}$. HD is due to the expansion of CAG triplet repeats in the Huntingtin (HTT) gene, leading to a polyglutamine tract in the N-terminal part of the protein $\mathrm{HTT}^{2}$. Both loss and gain of function of HTT contribute to the dysfunction and death of projection neurons in the caudate/putamen (striatum in mice) and cerebral cortex. A key neuropathological hallmark of HD is the presence of mutant HTT (mHTT) aggregates, primarily in neurons $s^{3}$, but also in glial cells ${ }^{4}, \underline{5}$. The brain of HD patients also displays reactive astrocytes, initially characterized by their hypertrophic morphology and overexpression of Glial Fibrillary Acidic Protein (GFAP) ${ }^{6-8}$. Astrocytes also display significant changes in gene expression in the putamen ${ }^{9}$ and cortex $\underline{5}$ of HD patients. Astrocytes are essential partners of neurons, as they perform many key functions including metabolic and trophic support, antioxidant defence and regulation of synaptic transmission and plasticity $\frac{10}{}$. How are these functions changed in the HD brain? Most studies report defective astrocyte functions in HD models $\frac{11}{11}$, including reduced glutamate uptake ${ }^{12}$, altered $\mathrm{K}^{+}$buffering ${ }^{13}$, impaired regulation of blood flow ${ }^{14}$, as well as reduced synthesis and release of antioxidants ${ }^{15}$, trophic factors $\frac{16}{}$, gliotransmitters ${ }^{17}$ and exosomes $\frac{18}{}$. However, in most cases, HD mouse models poorly replicate the reactive state of astrocytes observed in HD human brains, based on GFAP overexpression, hypertrophy $11, \underline{13}$ and transcriptional profile, as assessed recently with genome-wide transcriptomics, 19 , 20. Therefore, the impact of reactive astrocytes on disease progression is still unclear.

The Janus Kinase (JAK)-Signal Transducer and Activator of Transcription 3 (STAT3) pathway is a central pathway controlling astrocyte reactive response ${ }^{21}$. STAT3 is found activated in reactive astrocytes in genetic models of HD in mice and non-human primates $\frac{22}{}$, but it is still unknown whether this pathway is also activated in reactive astrocytes found in HD patients and which astrocyte functions are regulated by this pathway.

We previously reported that inhibition of the JAK2-STAT3 pathway in reactive astrocytes in an acute model of HD reduces their reactive features and increases the number of mHTT aggregates 22 . mHTT aggregates are mainly composed of N-terminal fragments of mHTT, which trap several important proteins such as transcription factors or chaperones $\underline{23}, \underline{24}$ and generate deleterious steric hindrance. However, aggregates may also contribute to remove toxic soluble mHTT from the cytosol ${ }^{25}$. Soluble mHTT can be degraded by the ubiquitin-proteasome system (UPS), while aggregates can only be cleared by autophagy coupled to lysosomal degradation $\underline{26}, 27$. Astrocytes are reported to have high proteolytic capacity, including for mHTT, which could explain why fewer aggregates are found in astrocytes than in neurons ${ }^{28}, \underline{29}$. Yet, it is unknown whether the UPS and autophagy/lysosomes systems are specifically altered in HD astrocytes ${ }^{30}, \underline{31}$, and how these systems can be stimulated in astrocytes to promote mHTT clearance. Another important proteostasis mechanism preventing mHTT aggregation is operated by chaperones, which promote HTT proper folding, prevent abnormal interactions with cellular proteins 
55 and guide mHTT to degradation systems ${ }^{32}$, 33 . In particular, Heat Shock Proteins (HSP) prevent mHTT 56 aggregation in different cell types $\underline{32}$, 34 .

57 Here, we studied how the JAK2-STAT3 pathway controls the reactive response of astrocytes in HD, 58 focusing on their ability to promote cellular proteostasis. We observed STAT3 activation in reactive 59 astrocytes in the brain of HD patients. In two HD mouse models, we found that activation of the JAK260 STAT3 pathway in reactive astrocytes reduces both the number and size of mHTT aggregates in neurons, without increasing soluble mHTT levels. Genome-wide transcriptomics and functional analysis

62 showed that JAK2-STAT3 pathway activation induces a specific proteostasis signature in astrocytes 63 associated with higher proteolytic activity. It also induces astrocyte expression of the co-chaperone 64 DNAJB1, which is loaded in exosomes and reduces mHTT aggregation in neurons. Our results show 65 that the JAK2-STAT3 pathway controls a bi-directional communication between reactive astrocytes and 66 neurons in HD, which eventually reduces mHTT aggregation and improves neuronal alterations. 


\section{Material and methods}

\section{Mice.}

Heterozygous knock-in mice (Hdh140 mice) expressing a chimeric mouse/human exon 1 containing $140 \mathrm{CAG}$ repeats inserted into the murine $\mathrm{Htt}$ gene on a C57BL/6J background were originally obtained

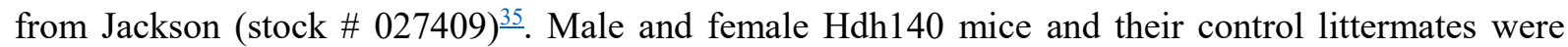
injected with different viral vectors (see below) at 7-10 months and euthanized 4 months later. Wild type (WT), adult male C57BL/6J mice were injected at 10 weeks of age, with different viral vectors and euthanized 6 weeks later.

All experimental protocols were reviewed and approved by the local ethics committee (CETEA $\mathrm{N}^{\circ} 44$ ) and the French Ministry of Education and Research (Approval APAFIS\#45542016031709173407). They were performed in an authorized animal facility (authorization \#D92-03202), in strict accordance with recommendations of the European Union (2010-63/EEC), and in compliance with the 3R guidelines. Animal care was supervised by a dedicated veterinarian and animal technicians. Mice were housed under standard environmental conditions (12-hour light-dark cycle, temperature: $22 \pm 1{ }^{\circ} \mathrm{C}$ and humidity: 50\%) with ad libitum access to food and water.

\section{Viral vectors.}

We either used lentiviral vectors (LV) or adeno-associated vectors (AAV) to drive transgene expression in neurons or in astrocytes, as described in each figure.

Self-inactivated (SIN) LV were produced by transient co-transfection of $293 \mathrm{~T}$ cells with four plasmids encoding viral structural proteins, the envelope protein and the transgenic cDNA under the control of a phosphoglycerate kinase 1 promoter (PGK1) and followed by the woodchuck hepatitis virus post-transcriptional regulatory Element (WPRE), to enhance transgene expression ${ }^{36}$. To target neurons, LV were pseudotyped with the G-protein of the vesicular stomatitis virus ${ }^{37}$. To target astrocytes, LV were pseudotyped with the rabies-related Mokola envelope and lentiviral recombinant genome contained four repeats of the miR124 target to repress transgene expression in neurons ${ }^{36}$. LV are referred to as " $\mathrm{LV}_{\mathrm{A}}$ - or $\mathrm{LV}_{\mathrm{N}}$-name of the transgene" depending the cell type targeted: A for astrocytes and $\mathrm{N}$ for neurons. Lentiviral particle titers were determined by ELISA quantification of the nucleocapsid p24 protein.

AAV (AAV2, serotype 9) contain the gfaABC1D promoter, a synthetic promoter derived from the GFAP promoter ${ }^{38}$, and transduce astrocytes. AAV were produced according to validated procedures ${ }^{39}$. Viral genome concentration was determined by qPCR on DNase resistant particles.

Viral vectors encoding murine SOCS3 or JAK2 ${ }^{\text {T875N }}$, a constitutively active form of JAK2 (JAK2ca), were used to respectively, inhibit or activate the JAK2-STAT3 pathway in mouse astrocytes ${ }^{22,}, 29,40$. They were co-injected with a viral vector encoding GFP or Td-Tomato to visualize transduced cells (same 
total viral load). Depending on the experiment, bilateral injections with the same viral vector were performed and controls were generated in different mice or the contralateral striatum was injected with the control viral vector encoding GFP only and data were analysed with paired statistical tests.

LV encoding the first $171 \mathrm{~N}$-terminal amino acids of human Huntingtin (HTT) cDNA with 82 polyglutamine repeats and that target either striatal neurons $\left[\mathrm{LV}_{\mathrm{N}}-\mathrm{mHTT}^{41}\right]$ or astrocytes $\left[\mathrm{LV}_{\mathrm{A}}-\mathrm{mHTT}^{8}\right]$ were used as LV-based models of HD.

Last, we generated $\mathrm{LV}_{\mathrm{A}}$ expressing full-length human DNAJB1 ( $\mathrm{LV}_{\mathrm{A}}-\mathrm{DNAJB} 1$ ) or the dominant negative mutant corresponding to the J-domain of human $D N A J B 1^{42}$ [ $\left.\mathrm{LV}_{\mathrm{A}}-\mathrm{DNAJB1}-\mathrm{DN}\right]$. The initial cDNA was generated by GeneArt Gene Synthesis services (Invitrogen, Carlsbad, CA) based on published sequences, and inserted into a $\mathrm{pENTR}^{\circledR}$ transfer plasmid. Gateway ${ }^{\circledR}$ recombination (Thermofisher Scientific) was used to clone these cDNA into appropriate SIN expression plasmid containing the PGK1 promoter, WPRE and four miR124 target sequences.

\section{Stereotactic injections.}

WT mice were anesthetized with an i.p. injection of ketamine $(100 \mathrm{mg} / \mathrm{kg})$ and xylazine $(10 \mathrm{mg} / \mathrm{kg})$. For Hdh140 mice, xylazine was replaced by medetomidine $(0.25 \mathrm{mg} / \mathrm{kg})$ and anaesthesia was reversed by a s.c. injection of atipamezole $(0.25 \mathrm{mg} / \mathrm{kg})$ at the end of the surgical procedure. Lidocaine $(7 \mathrm{mg} / \mathrm{kg})$ was injected subcutaneously at the incision site, $10 \mathrm{~min}$ prior to surgery. Mice were given paracetamol $(1.6 \mathrm{mg} / \mathrm{ml})$ in drinking water for $48 \mathrm{~h}$ after surgery. Viral vectors were injected in the striatum (coordinates from Bregma: anteroposterior: $+1 \mathrm{~mm}$, lateral: $+/-2 \mathrm{~mm}$; ventral: $-2.5 \mathrm{~mm}$ from the dura, with the tooth bar set at $0.0 \mathrm{~mm}$ ). LV were diluted in phosphate buffer saline (PBS) with $1 \%$ bovine serum albumin (BSA), at a total final concentration of $100 \mathrm{ng}$ p24/ $\mu$ l. AAV were diluted in $0.1 \mathrm{M}$ PBS with $0.001 \%$ pluronic acid, at a final total concentration of $2.5 * 10^{9}$ viral genome (VG)/ $/ \mu 1$. Diluted viral suspensions (2-3 $\mu 1$ depending on the cohort) were injected at a rate of $0.2-0.25 \mu \mathrm{l} / \mathrm{min}$ with a pump.

\section{Immunohistology.}

Mice were either killed with an overdose of sodium pentobarbital $(180 \mathrm{mg} / \mathrm{kg})$ and perfused with $4 \%$ paraformaldehyde (PFA) or by cervical dislocation and one brain hemisphere was rapidly dissected and drop-fixed in 4\% PFA. Brains were post-fixed for $24 \mathrm{~h}$ in $4 \%$ PFA, cryoprotected in $30 \%$ sucrose solution and cut on a freezing microtome into $30 \mu \mathrm{m}$-thick coronal sections. Series of brain sections were stored at $-20^{\circ} \mathrm{C}$ in an anti-freeze solution until used for immunostainings. 
Immunofluorescence. Brain sections were rinsed in PBS for $3 \times 10$ min and were blocked in $4.5 \%$ normal goat serum (NGS), $0.2 \%$ Triton X-100 in PBS (PBST) for $1 \mathrm{~h}$ at room temperature (RT). Brain sections were incubated overnight at $4{ }^{\circ} \mathrm{C}$ with the primary antibodies diluted in $3 \%$ NGS/PBST: anti-DARPP32647 (1:1,000, Mouse; Santa Cruz Biotechnology, Santa Cruz, CA, \#sc-271111 AF647), anti-GFAP-Cy3 (1:1,000, Mouse; Sigma, Saint-Louis, MO, \#C9205), anti-GFP biotinylated antibody (1:500, Goat; Vector Laboratories, Burlingame, CA, \#BA-0702), anti-IBA1 (1:500, Rabbit; Wako, Richmond, VA, \#019-19741), anti-MBP (1:500, Rabbit; Sigma, \#M3821), anti-Olig2 (1:500, Rabbit, Millipore, Burlington, MA, \#Ab9610), anti-S100ß (1:500, Mouse; Sigma \#S2532), anti-V5 (1:1,000, Mouse; Invitrogen, R96025), anti-Vimentin (1:1,000, Chicken; Abcam, Cambridge, UK, \#ab24525) and NeuroTrace 640/660 (1:250; Thermofisher Scientific, Waltham, MA, \#N21483). For DNAJB1 staining (1:100, Rabbit; Enzo Life Sciences, Farmingdale, NY, Hsp40/Hdj1 antibody ADI-SPA-400), brain sections were pretreated in $0.1 \mathrm{M}$ Tris- $\mathrm{HCl}, \mathrm{pH} 9$ at $95^{\circ} \mathrm{C}$ for $30 \mathrm{~min}$ and NGS was replaced by $5 \% \mathrm{BSA}$ in blocking solution and antibody diluent. For EM48 (1:200, Mouse; Merck, Kenilworth, NJ, MAB5374), sections were blocked in 3\% BSA, 2\% NGS/PBST and incubated with primary antibody in the same solution for $36 \mathrm{~h}$. STAT3 immunostaining was performed as described previously $\stackrel{22}{ }$. For NeuN staining (1:500, Mouse; Chemicon, Billerica, MA, \#MAB377), the "mouse on mouse" kit (Vector Laboratories) was used, according to the manufacturer's instructions. Brain sections were then rinsed 3 x 10 min in PBS and incubated with appropriate secondary Alexa Fluor-conjugated antibodies $(1: 1,000$, Goat; Invitrogen) or for GFP staining with Streptavidine-FITC $(1: 1,000$, Thermofisher Scientific, \#SA100-02) in 3\% NGS/PBST for $1 \mathrm{~h}$ at RT. Brain sections were rinsed three times with PBS before being mounted on SuperFrost ${ }^{\circledR}$ Plus slides (ThermoFisher Scientific) and coverslipped with Fluorsave $^{\mathrm{TM}}$ (Calbiochem, Darmstadt, Germany). Double or triple immunofluorescent stainings were performed successively, with each antibody incubated alone.

Immunohistochemistry with EM48 or Ubiquitin antibodies. Brain sections were pre-treated in $0.3 \%$ $\mathrm{H}_{2} \mathrm{O}_{2}$, blocked in $10 \%$ NGS/PBST (Ubiquitin) or 3\% BSA/PBST (EM48) and incubated overnight at $4^{\circ} \mathrm{C}$ in the same diluent, with primary antibodies directed against EM48 (1:200) or Ubiquitin $(1: 1,000$, Rabbit; Dako, Santa Clara, CA, z0458). After rinsing, brain sections were incubated with biotinylated secondary antibodies (1:1,000, Vector Laboratories) for $1 \mathrm{~h}$ at RT. Finally, they were incubated with the Vectastain Elite ABC Kit (Vector Laboratories) and revealed with the VIP kit (Vector Laboratories). Sections were rinsed three times with PBS before being mounted on SuperFrost ${ }^{\circledR}$ Plus slides, dehydrated and coverslipped with Eukitt (Sigma).

\section{Immunostaining quantification.}

The levels of GFAP immunoreactivity and the $\mathrm{GFAP}^{+}$volume were quantified on 10x-tiled images of serial sections along the antero-posterior axis of the striatum, acquired with an epifluorescence microscope equipped with a motorized stage (Leica DM6000, Nussloch, Germany). Virally transduced $\mathrm{GFP}^{+}$area was manually segmented on each section and the corresponding mean intensity signal for 
GFAP in this area was extracted with Image J. Background intensity signal was measured on unstained regions of the same section and subtracted to the GFAP total signal. The volume was calculated from the area measured on each section by the Cavalieri method $\underline{40}$.

To quantify lesion size, images of DARPP32 immunostained serial striatal sections were acquired at the $5 \mathrm{x}$ objective with an epifluorescence microscope (Leica DM6000). DARPP32-depleted area in the striatum was manually segmented with Image $\mathrm{J}$ on each serial section, and the total volume calculated with the Cavalieri method.

To quantify astrocyte soma area and STAT3 immunoreactivity, stacked confocal images of GFP and STAT3 immunostained sections were acquired with a $40 \times$ objective ( 3 brain sections per mouse, 3 fields per section, 10 to $16 \mathrm{z}$-steps of $1 \mu \mathrm{m}$, kept constant within a cohort, maximum intensity stack). $\mathrm{GFP}^{+}$ cell bodies were manually segmented and their individual area and mean grey value for STAT3 were measured with Image $\mathrm{J}$.

The total number and surface of $\mathrm{EM} 48^{+}$and $\mathrm{Ubiquitin}^{+}$aggregates were quantified on serial striatal sections along the antero-posterior axis, scanned with an Axio scanZ.1 (Zeiss, Oberkochen, Germany) at the 40x objective in bright field microscopy mode, with multi-plan focusing. Aggregates were automatically detected by the Morphostrider software (ExploraNova), with intensity, size and shape thresholds, after manual segmentation of the striatum on each section. The total number of aggregates within each striatum was then calculated. To quantify the distribution of EM48 ${ }^{+}$aggregates in DARPP $32^{+}$neurons and $\mathrm{GFP}^{+}$astrocytes, stacked confocal images (16 z-steps of $1 \mu \mathrm{m}$, maximum intensity stack) were acquired on a Leica TCS SP8 confocal fluorescent microscope with a 40x objective ( 3 brain sections per mouse, 3 fields per section). Aggregates were automatically detected with ImageJ software, with intensity, size and shape thresholds. Laser intensity, detection settings and analysis parameters were identical between each mouse of the same cohort. The number of aggregates in each cell type and the total number were manually quantified using ImageJ cell counter plugin.

\section{Staining of human brain sections.}

Frozen blocks of the putamen were obtained from the Netherland Brain Bank (NBB), Netherlands Institute for Neuroscience, Amsterdam (open access: www.brainbank.nl). All Material has been collected from donors from whom a written informed consent for a brain autopsy and the use of the material and clinical information for research purposes had been obtained by the NBB. Four HD patients (Vonsattel stage III) and four control subjects matched for age, sex and post-mortem delay were analysed (see Supplemental table 2). Blocks were cut into $10 \mu \mathrm{m}$ sections on a cryostat, post-fixed in 4\% PFA for $1 \mathrm{~h}$ and in ice-cold methanol for $10 \mathrm{~min}$. Sections were then incubated in $0.1 \mathrm{M}$ Tris- $\mathrm{HCl}(\mathrm{pH} 9)$ at $95^{\circ} \mathrm{C}$, for $20 \mathrm{~min}$, in $0.3 \% \mathrm{H}_{2} \mathrm{O}_{2}$ for $30 \mathrm{~min}$, and in 5\% BSA/PBST for $1 \mathrm{~h}$. Sections were incubated 48 $\mathrm{h}$ at $4{ }^{\circ} \mathrm{C}$ in $5 \% \mathrm{BSA} / \mathrm{PBST}$, with primary antibodies against GFAP $(1: 10,000$, Rabbit, Dako, \#Z0334292), STAT3 (1:200), NeuN (1:2,000) or DNAJB1 (1:100). After rinsing, sections were incubated with 
biotinylated secondary antibodies (1:1,000, Vector Laboratories) for $1 \mathrm{~h}$ at RT, and after rinsing, with the Vectastain Elite ABC Kit for $1 \mathrm{~h}$ and revealed with the DAB substrate kit (Vector Laboratories). Sections were incubated for $2 \mathrm{~s}$ in 50\% Mayer's hemalum solution (Merk, \#109249) and rinsed with tap water before being dehydrated and coverlslipped with Eukitt. Representative images in each panel were taken with a Leica microscope, in the same anatomical region identified on consecutive sections.

\section{Protein extraction.}

Mice were killed by cervical dislocation and their brains were rapidly collected. The striatum was dissected out on ice, snap frozen in liquid nitrogen and stored at $-80^{\circ} \mathrm{C}$ until protein extraction. Samples were homogenized by sonication in 20 volumes of lysis buffer [ $50 \mathrm{mM}$ Tris- $\mathrm{HCl} \mathrm{pH} 8,150 \mathrm{mM} \mathrm{NaCl}$, 1\% Triton X-100 (Tx) with 1:100 phosphatase inhibitors (Sigma, cocktail 2) and 1x protease inhibitors with EDTA (Roche, Basel, Switzerland)] and centrifuged at $16,000 \mathrm{~g}$ for $30 \mathrm{~min}$ at $4^{\circ} \mathrm{C}$. The supernatant contains Tx-soluble proteins and was used for immunoblotting. The Tx-insoluble pellet was washed with PBS and centrifuged at 16,000 g for $5 \mathrm{~min}$ at RT. The pellet was sonicated in a second lysis buffer [50 mM Tris-HCl pH 8, 2\% Sodium Dodecyl Sulfate (SDS) with 1:100 phosphatase and protease inhibitors and centrifuged at $16,000 \mathrm{~g}$ for $30 \mathrm{~min}$ at $4{ }^{\circ} \mathrm{C}$. The supernatant was collected and used for immunoblotting, as the SDS-soluble fraction.

\section{Exosome isolation.}

Mice were killed by cervical dislocation and their brains were rapidly collected to isolate exosomes, as described in Vella et al. ${ }^{43}$. The striatum was dissected out on ice, snap frozen in liquid nitrogen and stored at $-80^{\circ} \mathrm{C}$ until processing. Two or three striata were pooled by sample. Frozen striata were incubated in $75 \mathrm{U} / \mathrm{ml}$ collagenase III in Hibernate E solution (Thermofisher Scientific, $8 \mu \mathrm{l} / \mathrm{mg}$ ) at $37^{\circ} \mathrm{C}$ under agitation. After $5 \mathrm{~min}$, pieces of striatal tissue were gently pipetted up and down with a $1 \mathrm{ml}$ pipette, then with a large diameter fire-polished Pasteur pipette and incubated at $37^{\circ} \mathrm{C}$ for $10 \mathrm{~min}$. Then, tubes were gently inverted and returned to the incubation bath for $5 \mathrm{~min}$ (total incubation at $37^{\circ} \mathrm{C}: 20$ min). Tubes were collected on ice and protease inhibitors were added at $1 \mathrm{x}$ final concentration. To discard debris, samples were sequentially centrifuged at $4^{\circ} \mathrm{C}$ at $300 \mathrm{~g}$ for $5 \mathrm{~min}, 2,000 \mathrm{~g}$ for $10 \mathrm{~min}$ and $10,000 \mathrm{~g}$ for $30 \mathrm{~min}$. The last supernatants were collected and Hibernate $\mathrm{E}$ with protease inhibitors was added to a final volume of $3 \mathrm{ml}$. The first pellet was resuspended in $150 \mu \mathrm{l}$ of $50 \mathrm{mM}$ Tris- $\mathrm{HCl}, 1 \%$ SDS, $150 \mathrm{mM} \mathrm{NaCl}, 1 \mathrm{mM}$ EDTA, pH 7.4 with protease inhibitors, and sonicated to be analysed by immunoblotting as total brain homogenates. A sucrose step was prepared for each sample as $0.3 \mathrm{ml}$ of 2.5 M sucrose, $0.4 \mathrm{ml}$ of $1.3 \mathrm{M}$ sucrose, $0.4 \mathrm{ml}$ of $0.6 \mathrm{M}$ sucrose. The sample ( $3 \mathrm{ml}$ in Hibernate $\mathrm{E}$ ) was overlaid on top of the gradient. Sucrose steps were then centrifuged at $180,000 \mathrm{~g}$ for $190 \mathrm{~min}$ at $4^{\circ} \mathrm{C}$ in a SW60 swinging rotor (Beckman, Brea, CA). After removing the top $2.6 \mathrm{ml}$ of the step, three $0.4 \mathrm{ml}$ fractions (F1 to F3) were collected. Each fraction was diluted in $0.9 \mathrm{ml}$ cold Dulbecco's PBS (DPBS) with protease inhibitors and centrifuged at $100,000 \mathrm{~g}$ for $1 \mathrm{~h}$ at $4^{\circ} \mathrm{C}$ on a fixed TL1 10 rotor (Beckman). 
The pellet containing vesicles was resuspended in $10 \mu 1$ of cold DPBS with protease inhibitors and further diluted in loading buffer for immunoblotting or frozen until imaging by transmission electron microscopy (TEM).

The purity of exosomal fractions 2 and 3 was controlled by immunoblotting for known exosomal proteins (Flotillin-1 and TSG101) and for the mitochondrial protein voltage-dependent anion channel (VDAC), which is not present in exosomes (Supplemental Fig. 1c).

These fractions were also analysed by TEM as described previously ${ }^{44}$. Exosomal fractions were mixed with an equal volume of $4 \%$ PFA and incubated for $20 \mathrm{~min}$ at $4^{\circ} \mathrm{C}$. Fixed vesicles were then applied to carbon coated TEM grids and allowed to adsorb for 20 min at RT. TEM grids were then washed by sequential transfers on drops of PBS, incubated for 5 min at RT in $1 \%$ glutaraldehyde, and then washed by sequential transfers on drops of distilled water. Following negative-staining with $1 \%$ Gadolinium triacetate (EM stain 336, Agar Scientific, Stansted, UK) for $10 \mathrm{~min}$ at RT, samples were imaged in a Jeol 1400 transmission electron microscope (Jeol, Croissy/Seine, France). Images were recorded with a Gatan Orius CCD camera (Gatan, Pleasanton, CA) and processed with the Image J software.

\section{Immunoblot.}

Protein concentration was measured by the bicinchoninic acid assay (Pierce, Waltham, MA). Samples were diluted in loading buffer with dithiothreitol $\left(\mathrm{NuPAGE}^{\circledR}\right.$ LDS sample buffer and sample reducing agent, Invitrogen). Proteins (10 $\mu \mathrm{g}$ for Tx-soluble fraction and $5 \mu \mathrm{g}$ for SDS-soluble fraction) was loaded on a $7.5 \%$ or $4-12 \%$ Criterion $^{\mathrm{TM}}$ TGX Stain-Free Protein Gels (Bio-Rad, Hercules, CA). Protein concentrations in exosomal fractions were below detection level. Instead, an equal volume of each fraction was loaded on the gel. Migration was performed at $200 \mathrm{~V}$ for $30 \mathrm{~min}$ in Tris-glycine buffer (Bio-Rad) and proteins were transferred on a nitrocellulose membrane with the Trans-Blot Turbo ${ }^{\mathrm{TM}}$ Transfer System (Bio-Rad). After 3 x 10 min rinses in Tris Buffer Saline and 0.1\% Tween 20 (TBST), membranes were blocked in 5\% milk in TBST for $1 \mathrm{~h}$ at RT and incubated for $3 \mathrm{~h}$ at RT, or overnight at $4^{\circ} \mathrm{C}$ with the following primary antibodies: anti-Actin (1:5,000, Mouse; Sigma, \#A2066), antiFlotillin-1 (1:1,000, Rabbit; Sigma, F1180), anti-DNAJB1 (1:1,000), anti-HTT (2B4, 1:1,000, Mouse; Millipore, MAB5492), anti-mHTT (1C2, 1:1,000, Mouse; Millipore, MAB1574), anti-Ubiquitin (1:1,000), anti-TSG101 (1:500, Rabbit; Sigma, HPA006161), anti- $\alpha$-tubulin (1:5,000, Mouse; Sigma, T5168), and anti-VDAC (1:1,000, Rabbit; Abcam, ab15895). After 3 x 10 min washes in TBST, membranes were incubated for $1 \mathrm{~h}$ at RT with HRP-conjugated secondary antibodies (1:5,000, Vector laboratories) diluted in TBST with 5\% milk. Membranes were incubated with the Clarity Western ECL substrate (Bio-Rad) and the signal was detected with a Fusion FX7 camera (Thermofisher Scientific). Band intensity was quantified with Image $J$ and normalized to actin or $\alpha$-tubulin. For quantification of exosome proteins, the Stain-free technology (Bio-Rad) was used. Stain-free gels were exposed to UV light to activate tryptophan residues, resulting in UV-induced fluorescence of total loaded proteins. UV 
exposition and chemiluminescence acquisition were done with a ChemiDoc XRS+ system (Bio-Rad). DNAJB1 bands were normalized to total stained proteins using Image Lab Version 5.2.1 software (Bio$\mathrm{Rad})$. Each protein of interest was assessed at least on two different immunoblots.

\section{Quantification of cathepsin and proteasome activities in astrocytes.}

Hdh140 mice previously injected with astrocyte-specific AAV-GFP or AAV-Td-Tomato in the striatum to label astrocytes, were perfused with cold PBS for $4 \mathrm{~min}$. Their striata were rapidly collected in Hank's Balanced Salt Solution (HBSS; Sigma). Cells were mechanically and enzymatically dissociated with fire-polished Pasteur pipettes and the neural tissue dissociation kit with papain (Miltenyi Biotec, Bergisch Gladbach, Germany), following manufacturer's instructions. After filtration through a $50 \mu \mathrm{m}$-filter, myelin removal beads II and MS columns (Miltenyi Biotec) were used to deplete myelin from cell suspensions. Cells were then resuspended in 0.5\% PNB buffer (Perkin Elmer, FP1020) and incubated for $30 \mathrm{~min}$ with $1 \mu \mathrm{M}$ fluorescent cathepsin probe (iABP, Vergent Bioscience, Minneapolis, MN, \#40200,) or with $200 \mathrm{nM}$ proteasome probe (UbiQ, Bio BV, Amsterdam, the Netherlands, UbiQ-018,) at RT. Cells were centrifuged at $300 \mathrm{~g}$ for $5 \mathrm{~min}$ at $4{ }^{\circ} \mathrm{C}$ and resuspended in $400 \mu 1$ HBSS. They were sorted on a BD Influx cell sorter. GFP expressed by infected astrocytes was detected at 530/40 nm (488 nm excitation) and the cathepsin probe was detected at 670/30 nm (646 nm excitation). Td-Tomato expressed by infected astrocytes was detected at 579/34 nm (561 nm excitation) and the proteasome probe at 530/40 $\mathrm{nm}$ (488 nm excitation). Control samples of unlabelled or monofluorescent brain cells were used to define detector gains and sorting gates, which were kept constant for all samples. No compensation was required to accurately quantify the two fluorescent signals within the same cell. Cells were gated on a side scatter/ forward scatter plot, then singlets were selected and finally the percentage of $\mathrm{GFP}^{+} / \mathrm{Cathepsin}^{+}$or Td-Tomato ${ }^{+} /$Proteasome $^{+}$astrocytes was quantified in each mouse, after setting the gates on $\mathrm{GFP}^{+}$or $\mathrm{Td}^{-\mathrm{Tomato}^{+}}$astrocytes incubated without a probe (i.e. Fluorescence Minus One (FMO) controls, Supplemental Fig. 1b).

\section{RNA extraction on bulk samples and RT-qPCR.}

Mice were euthanized with an overdose of sodium pentobarbital, their brains were rapidly collected, the $\mathrm{GFP}^{+}$area was dissected out under a fluorescent macroscope (Leica) and lysed in Trizol with a MagNa Lyser instrument (Roche). Samples were stored at $-80^{\circ} \mathrm{C}$ until further processing. Samples were placed $5 \mathrm{~min}$ at RT before addition of chloroform for $3 \mathrm{~min}$, and centrifugation at 12,000 $\mathrm{g}$ for $15 \mathrm{~min}$ at RT. Aqueous phase was collected and 1 volume of $70 \%$ ethanol was added. Samples were transferred onto an RNeasyMin Elute spin column and RNA was purified according to manufacturer's instructions, with on-column DNase treatment (RNeasy micro kit, Qiagen, Hilden, Germany). RNA was eluted with $14 \mu \mathrm{l}$ of RNase-free deionized water and stored at $-80^{\circ} \mathrm{C}$ before transcriptomic analysis. RNA quality and integrity was evaluated with an Agilent RNA 6000 Pico assay and the Agilent 2100 Bioanalyzer (Agilent technologies, Santa Clara, CA). Reverse transcription was performed with the VILO ${ }^{\text {TM }}$ kit 
according to the manufacturer's protocol (SuperScript ${ }^{\mathbb{B}}$ VILO $^{\mathrm{TM}}$ cDNA synthesis kit; Life Technologies, Carlsbag, CA). Samples were diluted in $\mathrm{H}_{2} \mathrm{O}$ with $100 \mu \mathrm{g} / \mathrm{ml}$ BSA at $0.2 \mathrm{ng} / \mu 1$ and mixed with $250 \mathrm{nM}$ of each primers and iTaq Universal SYBR Green Supermix (Bio-rad) for qPCR. PCR efficiency was between 85 and 110\% for each set of primers (sequences shown in Supplemental table 1). Nucleasefree water and samples without reverse transcription were used as negative controls. Expression levels of transcripts of interest were normalized with the $\Delta \mathrm{Ct}$ method to the abundance of the best combination of normalizers among Eef1, Erp29, Ppia, Rpl13a, as identified with the Genorm method, implemented in Bio-rad CFX Manager software.

\section{Microarray analysis of acutely sorted astrocytes.}

WT mice injected with AAV-GFP or AAV-JAK2+AAV-GFP (same total viral load) were killed and their striatum rapidly collected in HBSS. The two striata of four mice were pooled before processing. Astrocytes were sorted as mentioned above, except that dissociated cells were prepared without the steps of myelin removal and probe incubation.

A total of 10 sorted cell samples were processed for microarray analysis $\left(4 \mathrm{GFP}^{+}\right.$astrocytes in the control WT-GFP group, $4 \mathrm{GFP}^{+}$astrocytes in the WT-JAK2ca group and $2 \mathrm{GFP}^{-}$cells in the WT-GFP group to validate astrocyte sorting efficiency). RNA was extracted as described in the previous paragraph. RNA quality and integrity was evaluated with an Agilent RNA 6000 Pico assay and the Agilent 2100 Bioanalyzer (Agilent technologies). RNA was amplified with the Ovation PicoSL VTA system V2 kit (NuGen technologies, San Carlos, CA). Single strand DNA and single primer isothermal amplification cDNA were purified with Agencourt RNAClean XP (NuGen technologies). cDNA concentration was measured with a Nanodrop-1000 spectrophotometer (Labtech France). The Encore biotiNL kit (NuGen technologies) was used for the fragmentation and labelling of the purified SPIA cDNA prior to hybridization on the Illumina BeadChip mouse WG-6v2, which contains more than 45,000 unique 50-mer oligonucleotides (Illumina, San Diego, CA). BeadChips were scanned on the Illumina Iscan. A control summary report was generated by the GenomeStudio software (Illumina) to evaluate the performance of built-in controls (variation in hybridization and background signals and background/noise ratio). Quantile normalization without background subtraction was applied to all samples within an analysis, with GenomeStudio software. First, to validate sorting efficiency, we compared the $4 \mathrm{GFP}^{+}$astrocyte samples with the $2 \mathrm{GFP}^{-}$cell samples from the WT-GFP group $(t$ test, $p$ $<0.05$ and Fold change $>1.5$ ). Then, we compared the $4 \mathrm{GFP}^{+}$astrocyte samples from WT-GFP mice with the $4 \mathrm{GFP}^{+}$astrocyte samples from the WT-JAK2ca mice to identify transcriptional changes induced by JAK2ca in astrocytes. To study only genes with reliable expression levels, we included probes with a "signal $p$ value" above 0.01 in more than $50 \%$ of the samples. Samples with a signal $p$ value below 0.01 were arbitrarily given a signal value of 75 (the lowest possible signal value being 76.5). Expression levels of detectable probes were compared between WT-GFP and WT-JAK2ca astrocytes with the limma (linear models for microarray data) package ${ }^{45}$. Analysis for enriched Gene 
ontology (GO) terms [Biological Processes (BP), Molecular Functions (MF) and Cellular Component (CC)] and Kyoto Encyclopaedia of Genes and Genomes (KEGG) pathways were performed with an adapted function from the R package limma (v3.44.3). Significant GO-BP and GO-MF entries relevant to proteostasis were selected, and all differentially expressed genes belonging to these GO entries were plotted as a network, with the 'cnetplot' function from the R package DOSE (v2.10.6). Microarray datasets are deposited on GEO under reference GSE107486.

\section{RNAseq analysis of acutely sorted astrocytes from Hdh140 mice.}

To analyse the transcriptome of astrocytes acutely sorted by FACS from the striatum of 12-14 monthold Hdh140 mice injected with AAV-GFP or AAV-JAK2ca (N=5-6), we followed the protocol described in Ceyzériat et al. $\stackrel{39}{ }$, except that the lower number of astrocytes sorted per sample was not compatible with RNA quality assessment, and full length double strand cDNA libraries were amplified with 16 LD-PCR cycles. The final mRNAseq libraries were then sequenced on a HiSeq 2500 Illumina platform $(2 \times 100 \mathrm{bp})$. Quality control of sequencing data was performed with FastQC (v0.11.9 $)^{46}$. Reads were mapped on the GRCm38 (mm10) mouse genome assembly with Hisat2 (v2.2.1) $)^{47}$. Quantification

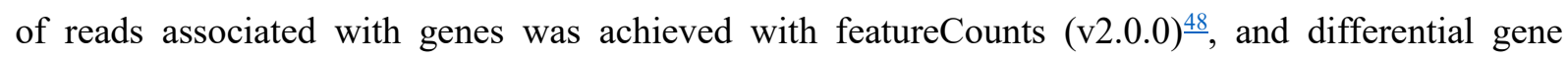
expression analysis was performed with DESeq2 (v1.28.1) on R (v4.0.2) ${ }^{49}$. Only genes with a raw number of counts $\geq 10$, in at least 3 samples were analysed. Results were considered statistically significant for an adjusted $p$ value $\leq 0.1$ and fold-changes $\geq 1.5$. GO and KEGG analysis was performed on $\mathrm{R}$ as described for microarray data, and fast preranked gene set enrichment analysis (GSEA) was carried out with the fgsea (v1.16.0) package on R as well卢. RNAseq data is deposited on GEO under reference GSE171141.

\section{Identification of regulatory transcription factors.}

We performed bioinformatics analysis on the list of 2,250 differentially expressed genes between control and HD astrocyte nuclei isolated from the cingulate cortex of human subjects (Additional file 9 in Al-Dalahmah et al. ${ }^{5}$ ). To identify putative upstream transcription factors, we used HOMER ${ }^{51}$ and Pscan with the TRANSFAC database ${ }^{52}$, two tools based on motif recognition in the promoter region of regulated genes. We also used three of the assembled transcription factor libraries from publicly available data (e.g. chromatin immunoprecipitation experiments, co-expression datasets) offered by the ChEA3 tool $\stackrel{53}{ }$ : Enrichr, ENCODE and ReMap.

\section{Statistics.}

Values for each individual samples are shown on graphs. Arithmetic means are represented by a horizontal line and paired samples from two groups are connected by a line. Sample size was chosen based on prior experience for each experiment, to yield adequate power to detect specific effects. Mice 
391 of the appropriate genotype were randomly allocated to experimental groups. Statistical analysis was

392 performed with Statistica software (StatSoft, Tulsa, OK) and graphs were prepared with GraphPad Prism

3937 (La Jolla, CA). Paired or unpaired two-tailed Student $t$-test were used to compare two groups, or

394 ANOVA to compare four groups. For each analysis, normality of residues and homoscedasticity were

395 assessed. If any condition of application was not fulfilled, we used non-parametric tests: two groups

396 were compared by the Mann-Whitney or Wilcoxon paired tests. Percentages were first changed to

397 proportions and transformed by the arcsine function, before being analysed by paired or unpaired $t$-test.

398 Investigators were partially blinded to the group when performing experiments and analysis, as the

399 group can be deduced by the presence of aggregates or GFP levels for example. The significance level

400 was set at $p<0.05$. Figure legends are encoded as follows $* p<0.05, * * p<0.01, * * * p<0.001$. In

401 each figure legend, $\mathrm{N}$ refers to the number of mice.

402 


\section{Results}

404

405

406

407

408

409

410

411

412

413

414

415

416

417

418

419

420

421

422

423

424

425

426

427

428

429

430

431

432

433

434

435

436

437

\section{STAT3 activation in reactive astrocytes in HD patients}

STAT3 is involved in the control of reactive astrocytes in multiple CNS diseases and their animal models $\frac{21}{}$, but it has never been studied in the brain of HD patients. We performed STAT3 immunostainings in the putamen of HD patients (Vonsattel grade $\mathrm{III}^{\underline{6}}$ ) and their age- and sex-matched controls. There was a stronger STAT3 immunoreactivity in HD patients, especially in regions showing neurodegeneration as seen by their lower density in $\mathrm{NeuN}^{+}$neurons and higher density in hypertrophic $\mathrm{GFAP}^{+}$astrocytes (Fig. 1). Many $\mathrm{STAT3}^{+}$cells had a typical astrocyte morphology and displayed nuclear accumulation of this transcription factor (Fig. 1), an indication of pathway activation ${ }^{21}$.

We also analysed available transcriptomic data of nuclei isolated from the cingulate cortex of grade III/IV HD patients ${ }^{\frac{5}{}}$ to identify potential active transcription factors in HD astrocytes. Several bioinformatics tools (based on literature mining or DNA motif recognition in the promoter of differentially expressed genes, see Methods) identified STAT3 as a potential regulatory transcription factor in HD astrocytes, with significant associated $p$ values (Table 1).

Together, these results support a role for STAT3 in driving astrocyte reactive changes in HD.

\section{Astrocytic JAK2-STAT3 pathway reduces neuronal mHTT aggregation in two HD mouse models}

To determine the molecular and functional regulation operated by STAT3 in HD reactive astrocytes, we took advantage of our viral vectors that transduce striatal astrocytes with high efficiency and selectivity (Supplemental Fig. 1a), to either activate or inhibit the JAK2-STAT3 pathway ${ }^{39}$, 40 .

We first studied knock-in Hdh140 mice that express a humanized HTT gene with 140 CAG repeats under its own endogenous promoter $\frac{35}{3}$. These mice develop progressive HD symptoms with small intraneuronal mHTT aggregates, early transcriptional defects in neurons, but very mild morphological and molecular reactive changes in astrocytes ${ }^{20}$, $\underline{54}$. In this model, we thus stimulated the JAK2-STAT3 pathway in striatal astrocytes by virus-mediated expression of a constitutively active form of JAK2 (JAK2ca).

A lentiviral vector targeting astrocytes and encoding JAK2ca ( $\left.\mathrm{LV}_{\mathrm{A}}-\mathrm{JAK} 2 \mathrm{ca}\right)$ was injected in 7-9 month-old heterozygous Hdh140 mice, with a $\mathrm{LV}_{\mathrm{A}}$ encoding GFP ( $\left.\mathrm{LV}_{\mathrm{A}}-\mathrm{GFP}\right)$ to visualize infected astrocytes (Hdh140-JAK2ca mice, Fig. 2a). Controls were Hdh140 mice injected with LV $\mathrm{A}_{\mathrm{A}}$ GFP at the same total viral titer (Hdh140-GFP mice), and brains from both groups were analysed 4 months later (Fig. 2a). Immunostaining on mouse brain sections showed that JAK2ca activated STAT3 (Fig. 2b) and induced the two cardinal features of reactive astrocytes: overexpression of the intermediate filaments GFAP (Fig. 2b, c) and soma hypertrophy (Fig. 2b, d). JAK2ca also increased mRNA levels of Vimentin and Serpina $3 n$, two markers of reactive astrocytes (Fig. 2e, f). We controlled that JAK2ca did not impact 
total mRNA levels of murine $H t t$ and $m H T T$, which were expressed at similar levels in Hdh140-JAK2ca and Hdh140-GFP groups ( $p=0.230$ and $p=0.258$ respectively, Student $t$-test).

JAK2ca expression in Hdh140 astrocytes significantly reduced the total number of mHTT aggregates in the striatum (Fig. 2g, h). The size of EM48 ${ }^{+}$aggregates was also significantly decreased in Hdh140JAK2ca mice (Fig. 2i). Most EM48 ${ }^{+}$aggregates were in small neuronal processes, while more than 30\% of them were found in the nucleus or cell body of striatal neurons expressing dopamine- and cAMPregulated neuronal phosphoprotein (DARPP32), and less than 1\% of all mHTT aggregates were found in $\mathrm{GFP}^{+}$astrocytes (Fig. 2j, k). Despite the marked reduction in the number of mHTT aggregates, this relative distribution was not significantly impacted by JAK2ca $\left(p=0.104\right.$ for DARPP32 ${ }^{+}$neurons, $p=$ 0.636 in $\mathrm{GFP}^{+}$astrocytes, $t$-test on arcsine-transformed data, Fig 2k).

To determine whether soluble mHTT was also reduced by JAK2ca, we analysed Triton X-100 (Tx)soluble protein extracts prepared from the striatum of WT and Hdh140 mice injected with $\mathrm{LV}_{\mathrm{A}}-\mathrm{GFP}$ or $\mathrm{LV}_{\mathrm{A}}-\mathrm{JAK} 2 \mathrm{ca}$. Immunoblotting with the $1 \mathrm{C} 2$ antibody that recognizes preferentially the elongated polyglutamine stretch of mHTT showed similar Tx-soluble mHTT levels in both groups (Fig. 2l, n, $p=$ 0.139 , paired $t$-test). We then immunoblotted proteins from the Tx-insoluble, SDS-soluble fraction with the 2B4 antibody, which preferentially binds to the N-terminal part of mHTT. High molecular weight forms of mHTT corresponding to aggregated fragments of mHTT were detected only in samples from Hdh140 mice. The levels of high molecular weight aggregated mHTT were lower in the Hdh140JAK2ca group, in accordance with histological data of reduced mHTT aggregation with JAK2ca (Fig. $2 \mathbf{m}, \mathbf{0})$.

Hdh140 mice do not show the typical striatal neurodegeneration observed in HD patients, but display early transcriptional defects in striatal neurons, in particular for Ppplrlb transcripts (which encodes the striatal protein DARPP32). In Hdh140 mice, JAK2ca expression in striatal astrocytes significantly increased Ppp1r1b mRNA levels (Fig. 2p), suggesting a beneficial effect upon neurons.

To provide an independent demonstration that JAK2-STAT3 pathway activation in striatal astrocytes reduces the amount and size of mHTT aggregates in neurons, we performed a complementary experiment by blocking this pathway in astrocytes, with its inhibitor Suppressor Of Cytokine Signalling 3 (SOCS3). For that, we selected another HD model that better replicates the strong neurodegeneration and subsequent astrocyte reactivity observed in the striatum of HD patients $\stackrel{8}{-22}$. This HD model involves lentivirus-mediated expression of the 171 first amino acids of HTT with 82 CAG repeats in striatal neurons ${ }^{41}$ (Fig 3a). In this model, SOCS3 expression in astrocytes efficiently blocked STAT3 activation and reactive changes in astrocytes (Fig $\mathbf{3 b}-\mathbf{f})^{22}$. SOCS3 expression in striatal astrocytes increased the aggregation of mHTT, as seen with EM48 immunostaining (Fig. 3g, h). Less than 2\% of mHTT aggregates were found in $\mathrm{GFP}^{+}$astrocytes (Fig. 3h), and this distribution was not changed by SOCS3 ( $p=0.380$, paired $t$-test on arsine-transformed data, data not shown). mHTT aggregates are ubiquitinated and the total number of Ubiquitin $(\mathrm{Ub})^{+}$aggregates was also increased by SOCS3 (Fig. 3i, 
j). In addition, aggregates were larger in the SOCS3 group than in the GFP group (Fig. 3k), revealing both quantitative and qualitative changes in neuronal mHTT aggregates following JAK2-STAT3 inhibition in astrocytes.

In the lentiviral HD model, mHTT causes local neuronal degeneration, visible as DARPP32-depleted lesion (Fig. 31). SOCS3 significantly increased the lesion volume (Fig 3m), and reduced Ppp1r1b mRNA levels (Fig. 3n).

Overall, our data show that activation of the JAK2-STAT3 pathway in reactive astrocytes reduces the number and size of neuronal mHTT aggregates and mitigates HD alterations, while blocking the pathway has opposite effects.

\section{JAK2ca regulates the expression of proteostasis genes in astrocytes}

How can JAK2-STAT3 pathway activation in reactive astrocytes impact mHTT aggregation in neurons? As the JAK2-STAT3 cascade regulates gene expression, we investigated transcriptional changes induced by JAK2ca by comparing the transcriptome of acutely sorted astrocytes isolated from WT-JAK2ca and WT-GFP control mice by microarray (Fig. 4a). Transduced astrocytes were collected by fluorescence-activated cell sorting (FACS) based on their GFP expression. GFP- cells, comprising microglia, neurons, oligodendrocyte precursor cells (OPC), oligodendrocytes and few non-infected astrocytes, were collected together. There were 1,415 differentially expressed transcripts (fold change $>1.5, p<0.05)$, between $\mathrm{GFP}^{+}$and GFP- cell samples in control WT-GFP mice (Fig. 4b). Besides $e G f p$, many known astrocyte gene markers were enriched in $\mathrm{GFP}^{+}$cells (e.g. AldoC, Aqp4, Gja 1, Gjb6, Slcla2, Slcla3). Conversely, known markers for microglial cells, neurons, OPC, oligodendrocytes were enriched in GFP- cells (e.g. Aif, Trem2, P2ry12, Pde10a, Snap25, Pdgfra, Myt1). The sorting procedure being validated, we next compared the gene expression profile of $\mathrm{GFP}^{+}$astrocytes isolated from WTGFP and WT-JAK2ca mice.

We found 888 probes (802 unique transcripts) differentially expressed between JAK2ca-astrocytes and control GFP-astrocytes, including Jak2 mRNA itself (Supplementary Fig. 2f). A Gene Ontology (GO) analysis revealed a significant enrichment in many GO-biological processes linked to immunity and inflammation, confirming that JAK2ca triggers reactive changes in astrocytes, which were also evidenced by morphological changes (Supplemental Fig. 2a-h) ${ }^{39}$.

Among the differentially expressed genes between GFP- and JAK2ca-astrocytes, there was a specific enrichment in biological processes linked to lysosomes and the UPS, as well as other processes related to proteostasis (Fig. 4c). KEGG pathway analysis also revealed a significant enrichment in the term "lysosome" in JAK2ca-reactive astrocytes $(p=0.0005)$. Several cathepsins (Ctsc, Ctss and Ctsz) were upregulated by JAK2ca in astrocytes (Fig. 4c). Genes linked to proteostasis formed a complex network of co-regulated genes in JAK2ca-astrocytes (Fig. 4c).

To confirm that the JAK2-STAT3 pathway was also able to induce a proteostasis gene signature in astrocytes in a HD context, we sorted striatal astrocytes from Hdh140-GFP and Hdh140-JAK2ca mice 
thanks to their GFP expression, and performed RNAseq analysis (Fig. 4d). Again, sorted astrocytes expressed high levels of astrocyte-specific genes and low or undetectable levels of known markers for microglia, neurons, cells of the oligodendrocyte lineage and endothelial cells (Supplemental Fig. 2i). Jak2 levels were significantly higher in JAK2ca-astrocytes than GFP-astrocytes (Supplemental Fig. 2j). Murine $H t t$, on the contrary, was expressed at low levels in sorted astrocytes from both groups (rpkm value $=1.859$ and 2.120 for GFP- and JAK2ca-astrocytes; adjusted $p$-value $=0.999)$, showing that JAK2ca does not change $H t t$ transcription in striatal astrocytes. We found 269 genes differentially expressed between Hdh140-GFP and Hdh140-JAK2ca astrocytes. Among them, many were linked to immunity/inflammation, as found in WT-JAK2ca astrocytes (data not shown). As observed in WTJAK2ca astrocytes, there was a significant enrichment in GO pathways related to proteostasis, including the molecular function "Heat shock protein binding" and the cellular components "lytic vacuole" and "lysosome" (Fig. 4e). Gene Set Enrichment Annotation (GSEA) also identified the term "phagosome", as significantly enriched in Hdh140-JAK2ca astrocytes, with a majority of up-regulated genes (normalized enrichment score $=1.759$, adjusted $p$ value $=0.039$ ).

Overall, this transcriptomic analysis shows that JAK2ca induces a specific proteostasis gene signature in striatal astrocytes both in WT and Hdh140 mice.

\section{JAK2ca increases proteolytic capacity in HD astrocytes}

It is important to establish that the identified transcriptional changes translate into detectable changes in astrocyte function ${ }^{55}$. To assess proteolytic activity of the two major clearance pathways in astrocytes, we used cell-permeable, activity probes for the lysosomal enzymes cathepsins and for the proteasome (Supplemental Fig. 1b).

We exposed acutely dissociated striatal cells from Hdh140-GFP and Hdh140-JAK2ca mice to a pancathepsin activity probe that becomes fluorescent when metabolized by cathepsins ${ }^{56}$. We then measured probe fluorescence in $\mathrm{GFP}^{+}$astrocytes from the two groups by FACS (Fig. 5a). There was a significantly larger fraction of $\mathrm{GFP}^{+}$astrocytes with high cathepsin activity in Hdh140-JAK2ca mice (Fig. 5b), revealing that JAK2ca increases lysosomal activity in astrocytes.

Another fluorescent probe was used to measure proteasome activity in acutely dissociated astrocytes (Fig. 5c) $)^{57}$. As the excitation/emission spectrum of this probe overlaps with GFP, we used a viral vector encoding the red fluorescent protein Td-Tomato instead of GFP to detect astrocytes in both groups. Again, the fraction of Td-Tomato ${ }^{+}$astrocytes with high proteasome activity was larger in Hdh140JAK2ca mice than in Hdh140-Td-Tomato mice (Fig. 5c), showing that reactive astrocytes also have a higher proteasome activity.

As several ubiquitin ligases were differentially expressed in JAK2ca-astrocytes (Fig. 4c), we assessed ubiquitination by immunoblotting striatal homogenates with a Ub antibody. We did not observe major changes in the pattern of Ub immunoreactivity or in total Ub levels between Hdh140-GFP and Hdh140-JAK2ca mice, both in Tx- and SDS-soluble fractions (Fig. 5d, e), suggesting that only the 
proteolytic step of the UPS is stimulated by JAK2ca, without global changes in the ubiquitination profile of mouse striatum.

To directly measure the intrinsic capacity of astrocytes to clear mHTT, we used viral vectors to force mHTT expression in astrocytes ( $\left.\mathrm{LV}_{\mathrm{A}}-\mathrm{mHTT}\right)$. WT mice were injected in the right striatum with $\mathrm{LV}_{\mathrm{A}^{-}}$ mHTT, $\mathrm{LV}_{\mathrm{A}}-\mathrm{SOCS} 3$, and $\mathrm{LV}_{\mathrm{A}}-\mathrm{GFP}$, and in the control left striatum with $\mathrm{LV}_{\mathrm{A}}-\mathrm{mHTT}$ and $\mathrm{LV}_{\mathrm{A}}-\mathrm{GFP}$, at the same total viral titer (Fig. 6a). Expression of mHTT in astrocytes also triggered STAT3 activation, as evidenced by its nuclear accumulation, and induced reactive changes in astrocytes (Fig. $\mathbf{6 b})^{8}$. In this model as well, SOCS3 efficiently reduced GFAP levels (Fig. 6b, c), STAT3 nuclear accumulation (Fig. $\mathbf{6 b}, \mathbf{d})$ and astrocyte soma hypertrophy (Fig. 6b, e). If the JAK2-STAT3 pathway enhances proteolytic activity in astrocytes, we reasoned that blocking this pathway with SOCS3 would increase mHTT aggregation in astrocytes. Indeed, we observed that the total number of mHTT aggregates in the striatum was increased with SOCS3 (Fig. 6f, g). Moreover, SOCS3 increased mHTT aggregate size (Fig. 6f, h). In this model, mHTT forms both nuclear and cytoplasmic inclusions in astrocytes (Fig. 6f). The fraction of nuclear aggregates in $\mathrm{GFP}^{+}$astrocytes was not changed by SOCS3 (19.1\% and 22.0\% respectively in GFP and SOCS3 groups, $p=0.458, t$-test on arcsine-transformed data, data not shown). These results show that the JAK2-STAT3 pathway stimulates astrocyte intrinsic capacity for mHTT clearance.

\section{JAK2ca induces chaperone expression in astrocytes}

Interestingly, several GO-molecular functions linked to chaperones and protein folding were significantly regulated by JAK2ca in astrocytes (Fig. $4 \mathbf{c}, \mathbf{e})$. Chaperones prevent mHTT aggregation ${ }^{32}$, $\underline{58}$ and can be released extracellularly in exosomes $\underline{59}$.

We focused on the co-chaperone DNAJB1 [DnaJ heat shock protein family (Hsp40) member B1], a member of the HSP40 family, which is induced nearly 3-fold by JAK2ca (Fig. 4c). DNAJB1 immunoreactivity was higher in the putamen of HD patients, specifically at the core of the degenerative area devoid of $\mathrm{NeuN}^{+}$neurons, where hypertrophic $\mathrm{GFAP}^{+}$astrocytes are abundant (Fig. 7a).

JAK2ca significantly increased DNAJB1 protein levels in the striatum of Hdh140 mice as seen by immunostaining (Fig 7b, d) and immunoblotting (Fig. 7c), while Dnajb1 mRNA were significantly reduced by SOCS3 in the lentiviral HD model (Fig. 7e). In Hdh140 mice, DNAJB1 displayed a diffuse cytosolic staining but also formed small nuclear inclusion-like structures, suggesting that DNJAB1 can be in close association with mHTT aggregates (Fig. 7d).

We next studied whether DNAJB1 was found in exosomes and whether DNAJB1 exosomal content was impacted in Hdh140-JAK2ca mice. Exosomal vesicles were isolated by biochemical fractionation from the striatum of Hdh140-GFP and Hdh140-JAK2ca mice, and they did contain DNAJB1 (Supplemental Fig. 1c). DNAJB1 normalized levels displayed a strong tendency to be higher in exosomes of Hdh140-JAK2ca mice than in Hdh140-GFP control mice ( $p=0.056$, Fig. 7f).

To assess whether DNAJB1 released by JAK2ca-astrocytes contributes to reduce mHTT aggregation in neurons, we generated viral vectors targeting astrocytes and encoding a dominant-negative form of 
human DNAJB1 (DNAJB1-DN), which prevents DNAJB1 interaction with the HSP70 chaperone ${ }^{42}$,

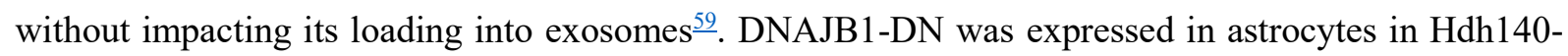
JAK2ca and Hdh140-GFP mice (Fig. 8a). DNAJB1-DN, detected by its V5 tag, was confirmed to be primarily expressed in striatal astrocytes (Fig. 8b). JAK2ca was still able to increase GFAP levels in Hdh140 astrocytes in presence of DNAJB1-DN (Fig. 8c). However, co-expression of DNAJB1-DN blocked JAK2ca-mediated reduction of EM48 ${ }^{+}$aggregate numbers (Fig. 8d) and even decreased Ppplrlb mRNA levels (Fig. 8e).

593 Conversely, we tested whether DNAJB1 restoration was able to oppose SOCS3 detrimental effects 594 in the LV model of mHTT overexpression. In the striatum of WT mice, we injected a viral vector 595 targeting astrocytes and encoding a full length human DNAJB1 ( $\left.\mathrm{LV}_{\mathrm{A}}-\mathrm{DNAJB} 1\right)$ with $\mathrm{LV}_{\mathrm{A}}-\mathrm{SOCS} 3$ or $596 \mathrm{LV}_{\mathrm{A}}$-GFP together with $\mathrm{LV}_{\mathrm{N}}-\mathrm{mHTT}$ (Fig. 8f). DNAJB1 did not interfere with SOCS3-mediated 597 reduction of GFAP levels in HD astrocytes (Fig. 8g). However, when DNAJB1 was expressed in 598 astrocytes, SOCS3 no longer exacerbated mHTT aggregation (Fig. 8h), neuronal lesion (Fig. 8i), or 599 reduced Ppplr 1 b mRNA levels (Fig. 8j), suggesting that DNAJB1 expression in astrocytes counteracts 600 SOCS3 deleterious effects.

601 Our results suggest that JAK2-STAT3 pathway-mediated induction of the co-chaperone DNAJB1 in 602 reactive astrocytes helps reduce mHTT aggregation and improve neuronal alterations in HD. 


\section{Discussion}

We studied how the JAK2-STAT3 pathway shapes the proteostasis response of reactive astrocytes in HD. We found that STAT3 is activated in reactive astrocytes of HD patients. Thanks to its targeted manipulation in striatal astrocytes of two complementary mouse models of $\mathrm{HD}$, we show that the JAK2STAT3 pathway controls the reactive state of astrocytes and reduces both the number and size of mHTT aggregates that form in neurons.

The reduction of mHTT aggregation by activation of the JAK2-STAT3 pathway in astrocytes is not due to lower mHTT expression, as Htt mRNA levels and Tx-soluble mHTT concentration were not reduced by JAK2ca. Importantly, JAK2ca-mediated reduction in mHTT aggregation did not trigger an increase in soluble mHTT levels either. This observation rules out the possibility that JAK2ca prevents mHTT from coalescing into aggregates or favours the accumulation of soluble mHTT after its dissociation from aggregates. Instead, activation of the JAK2-STAT3 pathway in reactive astrocytes appears to favour the full degradation of mHTT insoluble oligomers or aggregates, which could be mediated by autophagy-lysosomal removal of aggregates or chaperone-mediated extraction of mHTT and targeting to the UPS for complete clearance.

Indeed, transcriptomic analysis of acutely sorted astrocytes following JAK2-STAT3 pathway activation in WT and HD mice reveals extensive changes in genes linked to lysosomal degradation and the UPS. This result is in accordance with the single nuclei RNAseq (snRNAseq) analysis of astrocytes from the cingulate cortex of grade III/IV HD patients, reporting a significant enrichment in proteostasis functions ${ }^{\underline{5}}$. Lysosomes and UPS are active in all brain cells and changes in expression or activity in astrocytes could be masked by larger changes in other cell-types if assessed in typical bulk tissue analyses. We thus implemented two FACS-based assays to measure cathepsins and proteasome activity specifically in astrocytes. We found that JAK2ca increases both proteolytic activities in HD astrocytes. In addition, our ability to induce mHTT expression selectively in astrocytes by viral gene transfer provides a direct demonstration that the JAK2-STAT3 pathway increases reactive astrocyte capacity to clear mHTT.

As aggregates form mainly in neurons, trans-cellular signalling mechanisms must take place between neurons and reactive astrocytes (Fig. 9). Can mHTT be transferred from neurons to reactive astrocytes where they would be degraded more efficiently? In a landmark study in Drosophila, it was shown that mHTT exon 1 tagged with mCherry transfers from neurons to neighbouring phagocytic glia and forms aggregates with wildtype HTT in glia ${ }^{60}$. Other studies showed that mHTT can be exchanged between brain cells in Drosophila, in mice ${ }^{61-63}$, and even in humans as mHTT aggregates were detected in healthy embryonic neurons grafted in the brain of HD patients ${ }^{64}$. mHTT can be packaged in exosomes of different cell types ${ }^{65}, \underline{66}$ and be taken up by neighbouring cells, including neurons. Exchange of mHTT may also involve direct cell-to-cell contacts via tunnelling nanotubes ${ }^{67}$ or unconventional secretory pathways ${ }^{68}$. Most studies were performed in vitro or in non-neuronal cells, therefore, the precise 
mechanisms of mHTT exchange from neurons to astrocytes in the mammalian brain remain to be elucidated. Of note, a recent study showed that astrocyte-specific silencing of mHTT in a genetic HD mouse model reduces both astrocyte and neuronal mHTT aggregates $\frac{20}{}$, further supporting the concept of a tight partnership between these two cell types to degrade mHTT.

An alternative and non-exclusive mechanism for reduced neuronal mHTT aggregation upon JAK2STAT3 pathway activation in astrocytes, is that reactive astrocytes release proteins that promote mHTT clearance within neurons (Fig. 9). Our transcriptomic study shows that several chaperones are induced by JAK2ca in reactive astrocytes. In particular, DNAJB1 protein levels were higher in Hdh140-JAK2ca mice and this co-chaperone was abundant in extracellular exosomes. Chaperones are known to be released in exosomes and mediate trans-cellular proteostasis ${ }^{34}, \underline{59}$. Moreover, exosomes isolated from cultured astrocytes were shown to reduce mHTT aggregation in HD mice ${ }^{18}$. Interestingly, snRNASeq shows that $D N A J B 1$, as well as other chaperones are significantly overexpressed in astrocytes from the cingulate cortex of grade III/IV HD patients $\underline{\underline{5}}^{-}$and the putamen of grade II/III patients ${ }^{9}$, supporting that this beneficial proteostasis response also occurs in astrocytes from HD patients.

Through its J domain, DNAJB1 interacts with HSP70 to stimulate its ATP-dependent chaperone activity ${ }^{69}$. This domain is also implicated in DNAJB1 loading into exosomes ${ }^{42}, \underline{59}$. The $\mathrm{J}$ domain alone cannot activate HSP70 and has a dominant-negative action on the endogenous DNAJB1 ${ }^{42}$. Expression of this mutant in astrocytes abrogated JAK2ca-mediated beneficial effects in Hdh140 mice, showing DNAJB1 involvement in JAK2ca effects. Conversely, expression of DNAJB1 in astrocytes cancelled SOCS3 deleterious effects on neuronal death and transcriptional defects in the lentiviral HD model. Overall, our data strongly suggest that DNAJB1, produced by reactive astrocytes following JAK2STAT3 pathway activation, contributes to mHTT clearance.

DNAJB1 was shown to be the rate-limiting chaperone to suppress aggregation of a short fragment of $\mathrm{mHtt}^{70}$. But interestingly, HSPs not only prevent mHTT aggregation but can also promote solubility of proteins trapped in aggregates like transcription factors and favour mHTT degradation by addressing it to the UPS or autophagy-lysosomes ${ }^{33}$. Of note, DNAJB1 itself was recently shown to promote $\alpha$ synuclein disaggregation ${ }^{71}$. HSP-mediated extraction of housekeeping proteins or mHTT itself from aggregates is expected to reduce their size, which is consistent with our observations. Therefore, HSP have multiple actions that can in fine protect neurons against mHTT toxicity, as shown in different experimental systems based on HSP overexpression ${ }^{72-74}$. Here, we show that neurons rely on the endogenous production of chaperones by astrocytes to reduce mHTT aggregation.

The toxicity of mHTT aggregates is still discussed ${ }^{24,75}$ and $\mathrm{mHTT}$ aggregates could have a biphasic action ${ }^{76}$. At early stages, they could trap soluble toxic mHTT and prevent its deleterious interaction with key cellular partners. Later, mHTT aggregates could be detrimental by sequestering transcription factors, housekeeping proteins or microRNAs, leading to neuronal dysfunction and necrotic death 24,77 . It is important to note that most of these studies were based on in vitro systems allowing time-lapse monitoring of aggregates, but which cannot fully replicate the complex brain environment where 
677 neurons interact with multiple glial cells and have to cope with mHTT for months, and even decades in

678 patients. Here, in two complementary HD mouse models, we report that reduced mHTT aggregation is

679 associated with improved neuronal features, showing that the JAK2-STAT3 pathway shapes a beneficial

680 reactive response in striatal astrocytes. It will be important to further explore how this pathway

681 specifically regulates other key astrocyte functions such as glutamate uptake or potassium buffering,

682 which were shown to be altered in HD models and patients ${ }^{13}$.

683

684 In conclusion, we show that the JAK2-STAT3 pathway activates a beneficial proteostasis program 685 in reactive astrocytes, which helps neurons handle toxic mHTT. Our study uncovers two non-mutually 686 exclusive, bi-cellular mechanisms to reduce mHTT aggregation in HD neurons (Fig. 9): one relying on 687 mHTT exchange and clearance within reactive astrocytes and the other involving the release of 688 chaperones from reactive astrocytes to promote neuronal proteostasis. Astrocytes are not only defective 689 in HD as usually reported, they may also acquire enhanced capacities to promote mHTT clearance and 690 neuronal function, following activation of specific signalling cascades. Our results open new therapeutic 691 avenues to further enhance the natural partnership between reactive astrocytes and vulnerable neurons 692 in HD.

693 
694

695

696

697

698

699

700

701

702

703

704

705

706

707

708

709

710

711

712

713

714

715

716

717

718

719

720

721

\section{Acknowledgments}

This study was supported by CEA, CNRS and grants from the French National Research Agency (grants \# 2010-JCJC-1402-1, 2011-BSV4-021-03, ANR-16-TERC-0016-01 and ANR-20-CE16-0012-02 to C.E., 2011-INBS-0011 for NeurATRIS national infrastructure to P.H., as well as the EpiHD project ANR-17-CE12-0027 and a grant from H2020 ERA-Net for Research Programs on Rare Diseases (TreatPolyQ project ANR-17-RAR3-0008-01) to E.B and from Fondation maladies rares (GenOmic_2019-0203, Program High throughput sequencing and rare diseases) to C.E. C.E. and L.A. received support from the Association Huntington France. L.A. holds a PhD fellowship from the Region Ile-de-France via the DIM Cerveau et Pensée. Sequencing was performed on a platform partially supported by the France Génomique national infrastructure, funded by the Investissements d'Avenir program managed by the French National Research Agency (ANR-10-INBS-09). The present work also benefited from Imagerie-Gif core facility supported by French National Research Agency (ANR-11EQPX-0029/Morphoscope, ANR-10-INBS-04/FranceBioImaging; ANR-11-IDEX-0003-02/ Saclay Plant Sciences).

We are grateful to F. Aubry for help with initial AAV vector cloning and FACS experiments, C. Joséphine for AAV production, G. Auregan for stereotactic surgery and Dr. J. Baijer and N. Dechamps for FACS-isolation of astrocytes. We thank Prof. Déglon for helpful discussions at the beginning of the project. We acknowledge the help of V. Lavilla, and Cédric Fund on transcriptomic studies at the CNRGH. We thank Drs. K. Cambon and G. Liot for sharing their antibodies to S100ß, VDAC and mHTT. We thank L. de Longprez for help with the Hdh140 colony, as well as Dr. N. Heck, E. Saavedra and R. Jacqmin for pilot experiments.

\section{Author contribution}

LA, CE: conception; LA, LBH, MACS, CE: design of the work; LA, LBH, PG, MACS, CD, MAP, FP, ASH, MG, MGG, MK: acquisition of data; LA, LBH, MRP, PG, NS, NR, PdlG, RO: analysis of data; LA, LBH, MRP, EBo, SB, RO, EBr, MACS, CE: interpretation of data; MCG, ND, RM, APB, GB: Provided reagents or materials; JFD, PH, EBr, CE: Provided funding; LA, CE: manuscript writing. All authors revised and approved the manuscript. 


\section{Figures}
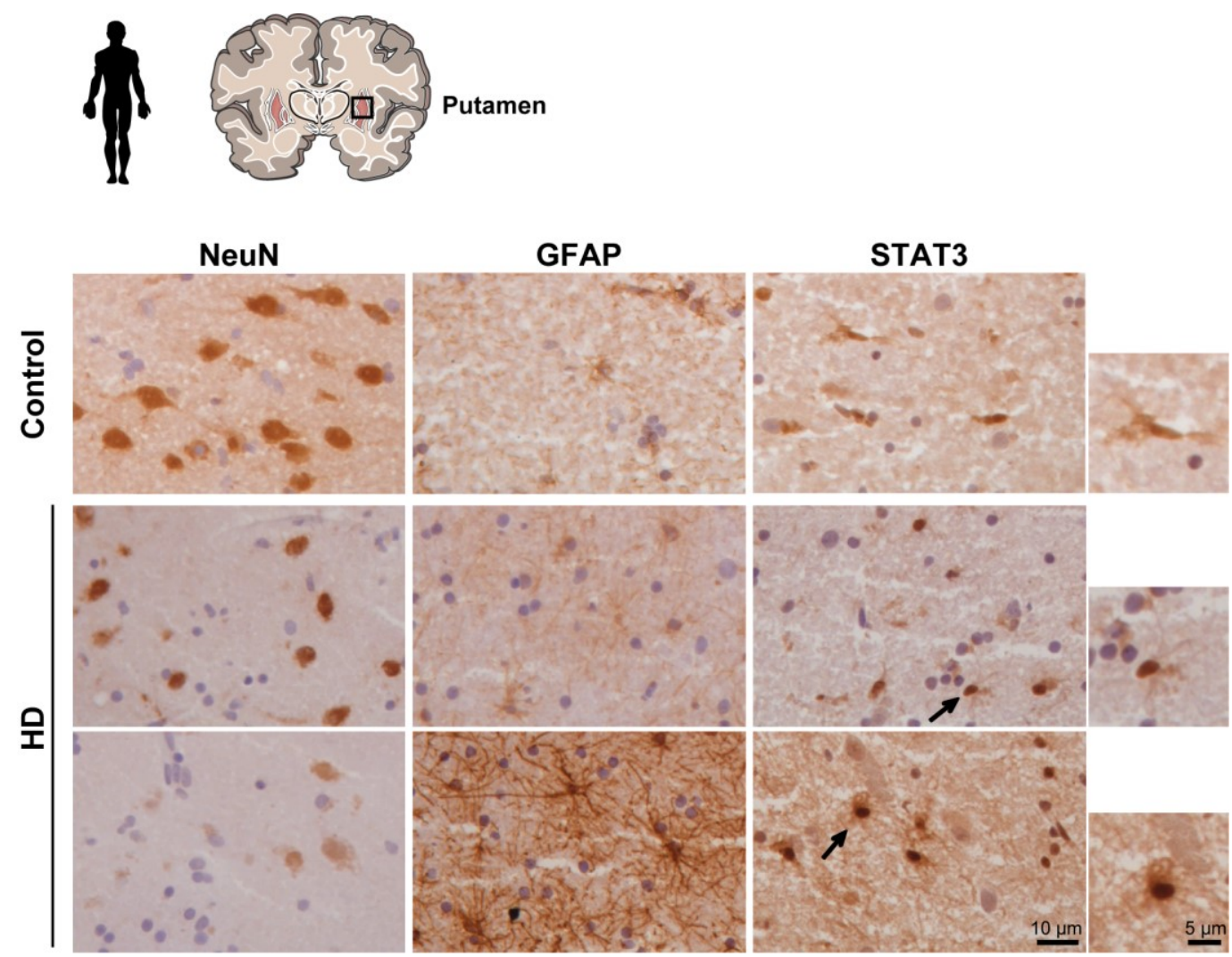

\section{Figure 1. STAT3 nuclear accumulation in the putamen of HD patients}

724 STAT3 immunoreactivity is higher in the putamen of HD patients than in control subjects, especially in

725 regions displaying many hypertrophic $\mathrm{GFAP}^{+}$astrocytes, and major neurodegeneration, as seen with the loss NeuN staining ( $3^{\text {rd }}$ line). STAT3 is often found accumulated in the nucleus of cells with a typical astrocyte morphology (arrows and high magnification). Representative images from 4 subjects/group. 

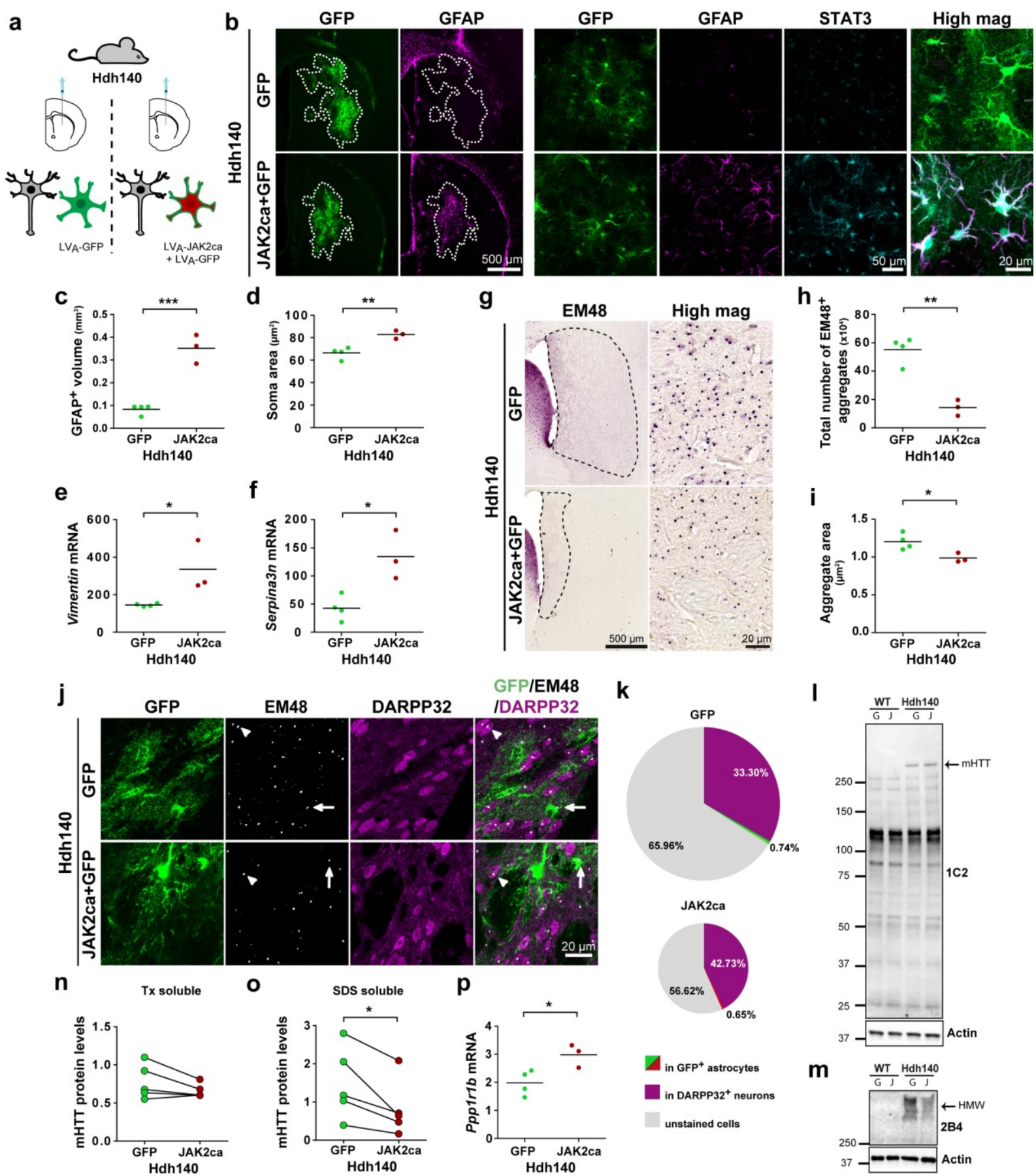

\section{$\mathbf{k}$}
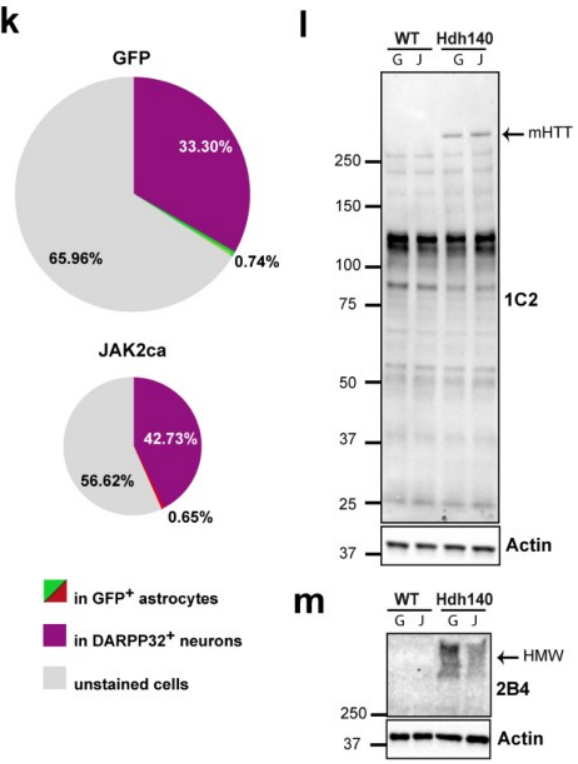 \\ Figure 2. JAK2ca induces astrocyte reactivity and reduces neuronal mHTT}

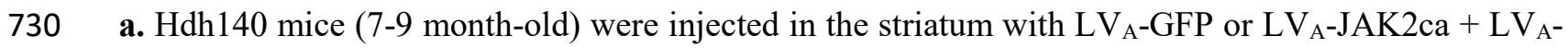

731 GFP, at the same total virus load, and their brains analysed 4 months later. b. Low magnification images

732 (left) show the $\mathrm{GFP}^{+}$transduced area (outlined, green) and GFAP staining (magenta) in the striatum of

733 Hdh140-GFP and Hdh140-JAK2ca mice. High magnification images (right) of astrocytes stained for

734 GFP (green), GFAP (magenta), and STAT3 (cyan). JAK2ca triggers STAT3 activation, as seen by its

735 nuclear accumulation, increases GFAP and vimentin levels and induces morphological changes in 
astrocytes. Note that the basal expression of GFAP is nearly undetectable in Hdh140 mice, suggesting very mild reactive changes in this model. $\mathbf{c}, \mathbf{d}$. $\mathrm{GFAP}^{+}$volume $(\mathbf{c})$ and soma area of $\mathrm{GFP}^{+}$astrocytes $(\mathbf{d})$ are significantly increased by JAK2ca. e, f. JAK2ca increases Vimentin (e) and Serpina3n (f) mRNA levels. g. Bright field images of EM48 ${ }^{+}$aggregates in the striatum of Hdh140-GFP and Hdh140-JAK2ca mice. The striatal region displaying EM48 ${ }^{+}$aggregates is outlined on low magnification images. $\mathbf{h}, \mathbf{i}$. Total number (h) and size (i) of EM $48^{+}$aggregates are significantly decreased by JAK2ca in the striatum of Hdh140 mice. j. Confocal images of striatal sections stained for GFP (green), EM48 (white) and

743 DARPP32 (magenta). EM48 ${ }^{+}$aggregates are mostly found in neurons labelled with DARPP32

744 (arrowhead) and very rarely in $\mathrm{GFP}^{+}$astrocytes (green, arrow). k. JAK2ca decreases the total number 745 of EM48 $8^{+}$aggregates, but the distribution of EM48 ${ }^{+}$aggregates between $\mathrm{GFP}^{+}$astrocytes and 746 DARPP32 ${ }^{+}$neurons is not changed. $\mathbf{~}, \mathbf{n}$. Immunoblotting on the Tx-soluble fraction of mHTT with the 747 1C2 antibody. Similar levels of mHTT are detected in Hdh140-GFP and Hdh140-JAK2ca mice, while mHTT is undetectable in WT mice. There is no mHTT cleavage fragments detected by this antibody in both Hdh140-GFP and Hdh140-JAK2ca mice. m, o. Immunoblotting on the SDS-soluble fraction of high molecular weight species (HMW) of mHTT with the 2B4 antibody. JAK2ca decreases the levels of insoluble HMW mHTT species in Hdh140 mice. Band intensity was normalized to actin. p. Striatal Ppp1r1b mRNA levels are higher in Hdh140-JAK2ca mice than in Hdh140-GFP mice. c-f, h, i, p. Student $t$-test. $\mathrm{N}=3-5 /$ group. $\mathbf{n}, \mathbf{o}$. Paired $t$-test. $\mathrm{N}=5$ /group. 
bioRxiv preprint doi: https://doi.org/10.1101/2021.04.29.441924; this version posted April 29, 2021. The copyright holder for this preprint (which was not certified by peer review) is the author/funder, who has granted bioRxiv a license to display the preprint in perpetuity. It is made available under aCC-BY-NC-ND 4.0 International license.

a

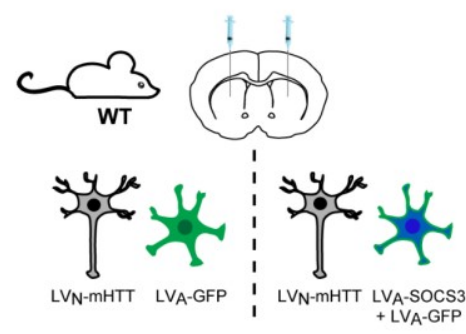

b

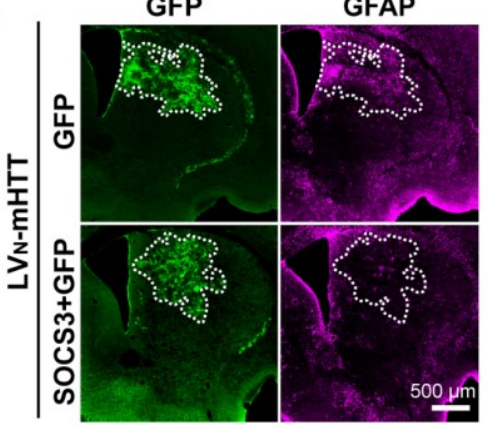

$500 \mu \mathrm{m}$

GFPIGFAP

ISTAT3

High mag

STAT3

C

GFP

GFAP
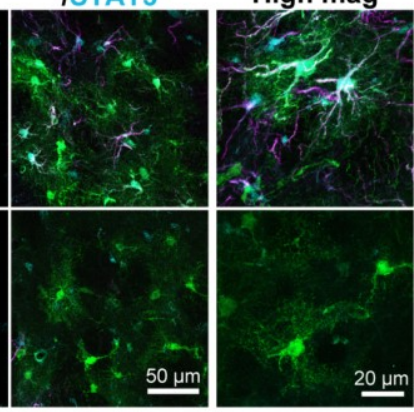

d
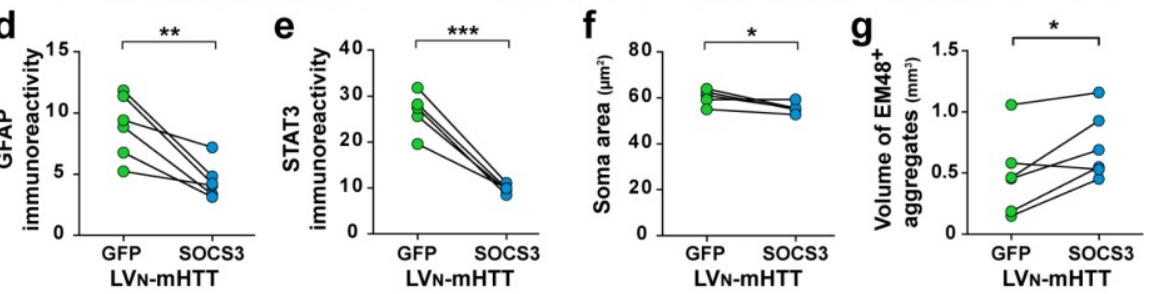

h

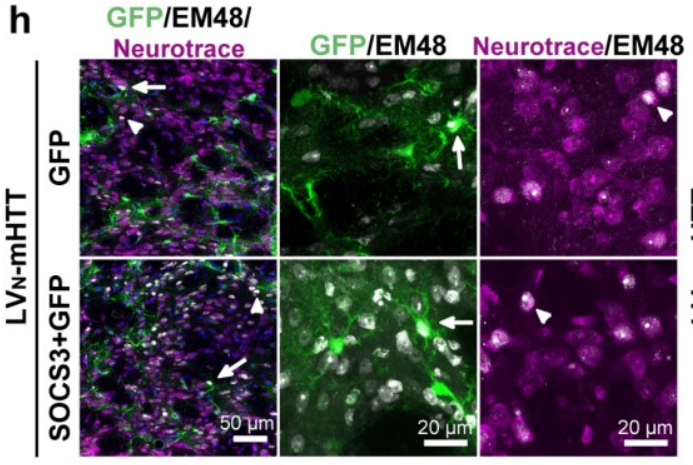

i
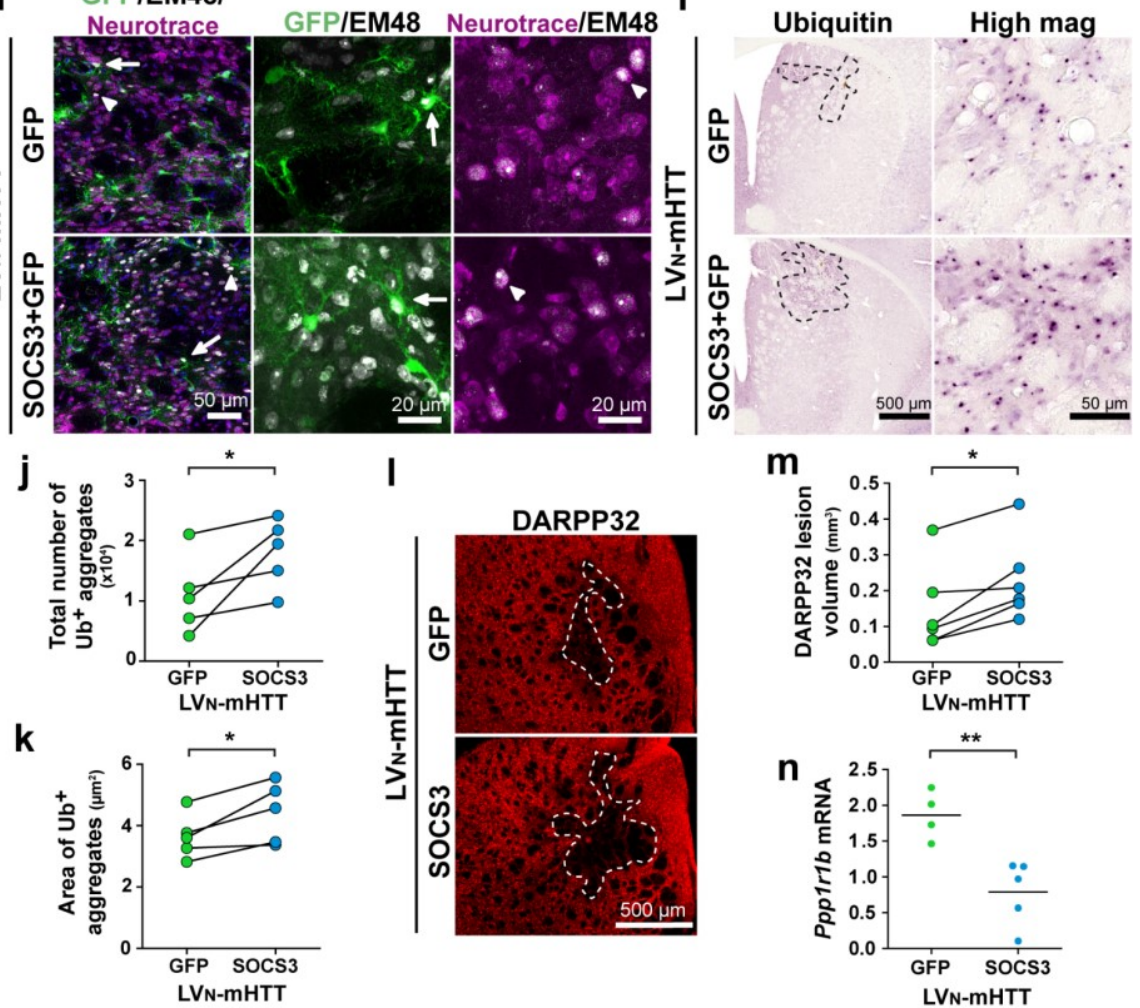


\section{Figure 3. SOCS3-inhibition of the JAK2-STAT3 pathway in astrocytes increases}

\section{5 the number and size of neuronal mHTT aggregates}

756 a. Two month-old WT mice were injected with $\mathrm{LV}_{\mathrm{N}}-\mathrm{mHTT}+\mathrm{LV}_{\mathrm{A}^{-}}-\mathrm{GFP}$ in one striatum and with $\mathrm{LV}_{\mathrm{N}^{-}}$

$757 \mathrm{mHTT}+\mathrm{LV}_{\mathrm{A}}-\mathrm{SOCS} 3+\mathrm{LV}_{\mathrm{A}}-\mathrm{GFP}$ in the contralateral striatum, at the same total virus load. Their brains

758 were analysed 6 weeks later. b. Low magnification images show the transduced $\mathrm{GFP}^{+}$area (green,

759 outlined) in the striatum and immunostaining for GFAP (magenta). c. High magnification confocal 760 images of astrocytes stained for GFP (green), GFAP (magenta) and STAT3 (cyan). d-f.

761 Immunoreactivity for GFAP (d) and STAT3 (e), as well as astrocyte soma area (f) are significantly 762 decreased by SOCS3. g. The striatal volume with EM48 ${ }^{+}$aggregates is significantly increased by 763 SOCS3. h. Confocal images of striatal sections stained for GFP (green), EM48 (white), neurotrace 764 (magenta) and DAPI (blue). Large EM48 ${ }^{+}$aggregates of mHTT are mostly found in neurons stained for 765 neurotrace, occupying their entire nucleus (arrowhead). Only few $\mathrm{GFP}^{+}$astrocytes display an EM48 ${ }^{+}$ 766 aggregate (arrow). i. Immunolabeling for ubiquitin (Ub) shows $\mathrm{Ub}^{+}$aggregates in the striatum (delimited 767 with black dots). j. The number of $\mathrm{Ub}^{+}$inclusions is significantly increased by SOCS3. k. $\mathrm{Ub}^{+}$inclusions 768 are larger in the striatum injected with $L V_{A}-S O C S 3$ than $L_{A}-G F P$. l. Images of striatal sections stained 769 for DARPP32 (red) showing the striatal lesion caused by mHTT (dotted lines). m. Striatal DARPP32770 lesions are significantly larger in the SOCS3 group. n. SOCS3 decreases mRNA levels of the neuronal 771 transcripts Ppp1r1b (Darpp32). d-g, k. Paired $t$-test. N = 5-6/group. j, m. Wilcoxon paired test. $\mathrm{N}=5$ 772 6/group. n. Student $t$-test. $\mathrm{N}=4$-5/group. 


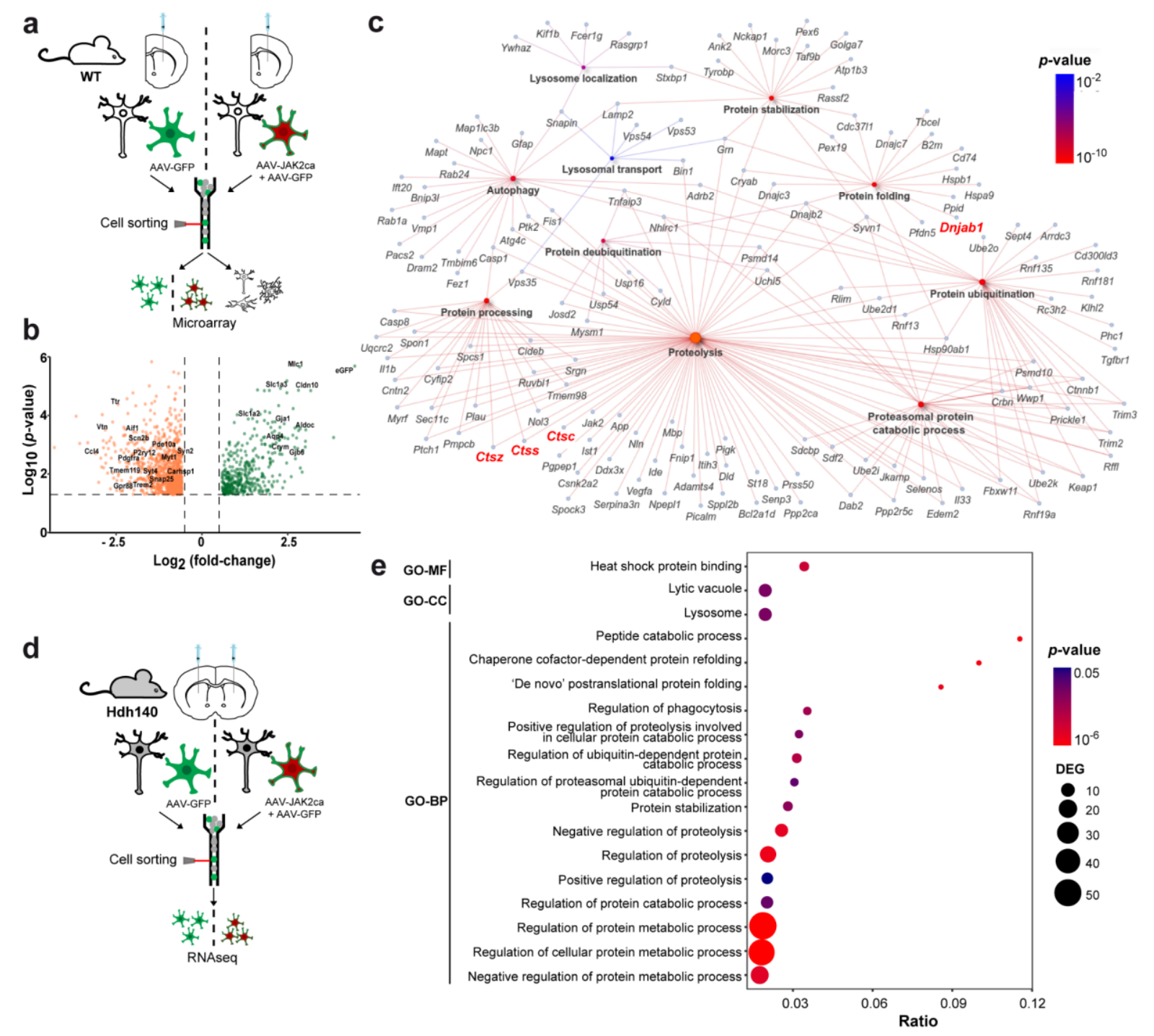

773 Figure 4. JAK2ca regulates the expression of proteostasis genes in astrocytes

774 a. Two month-old WT mice were injected in the striatum with AAV-GFP or AAV-JAK2ca + AAVGFP ( $\mathrm{N}=4$ /group), at the same total virus load. After 2 months, $\mathrm{GFP}^{+}$striatal astrocytes were acutely sorted and their transcriptome analysed by microarray. b. Validation of astrocyte isolation. The volcano plot shows the 1,415 differentially expressed genes between $\mathrm{GFP}^{+}$astrocytes and $\mathrm{GFP}^{-}$cells in WT-GFP mice (in green overexpressed in $\mathrm{GFP}^{+}$cells; in orange, overexpressed in $\mathrm{GFP}^{-}$cells). eGFP and established cell-type specific markers are shown. $\mathrm{GFP}^{+}$cells express typical astrocyte markers while GFP- $^{-}$cells express markers for microglial cells, neurons, cells of the oligodendrocyte lineage and endothelial cells. c. GO analysis on the list of JAK2ca-regulated genes in $\mathrm{GFP}^{+}$astrocytes reveals a significant enrichment in several biological processes linked to autophagy/lysosome or UPS and in molecular functions linked to chaperones. Network plot with the genes involved in the selected GO pathways. d. Hdh140 mice (8-10 month-old) were injected in the striatum with AAV-GFP or AAVJAK2ca + AAV-GFP, at the same total virus load. After 4 months, $\mathrm{GFP}^{+}$striatal astrocytes were collected and analysed by RNAseq. e. GO analysis reveals a significant enrichment in genes involved in molecular functions (MF), cellular components (CC) and biological processes (BP) linked to 
bioRxiv preprint doi: https://doi.org/10.1101/2021.04.29.441924; this version posted April 29, 2021. The copyright holder for this preprint (which was not certified by peer review) is the author/funder, who has granted bioRxiv a license to display the preprint in perpetuity. It is made available under aCC-BY-NC-ND 4.0 International license.

proteostasis. The $\mathrm{x}$ axis represents the ratio of the number of differentially expressed genes over the total number of genes belonging to a $G O$ entry, $D E G=$ differentially expressed genes. $\mathbf{b}-\mathbf{c} . \mathrm{N}=4$ /group. e. $\mathrm{N}=5$-6/group. 

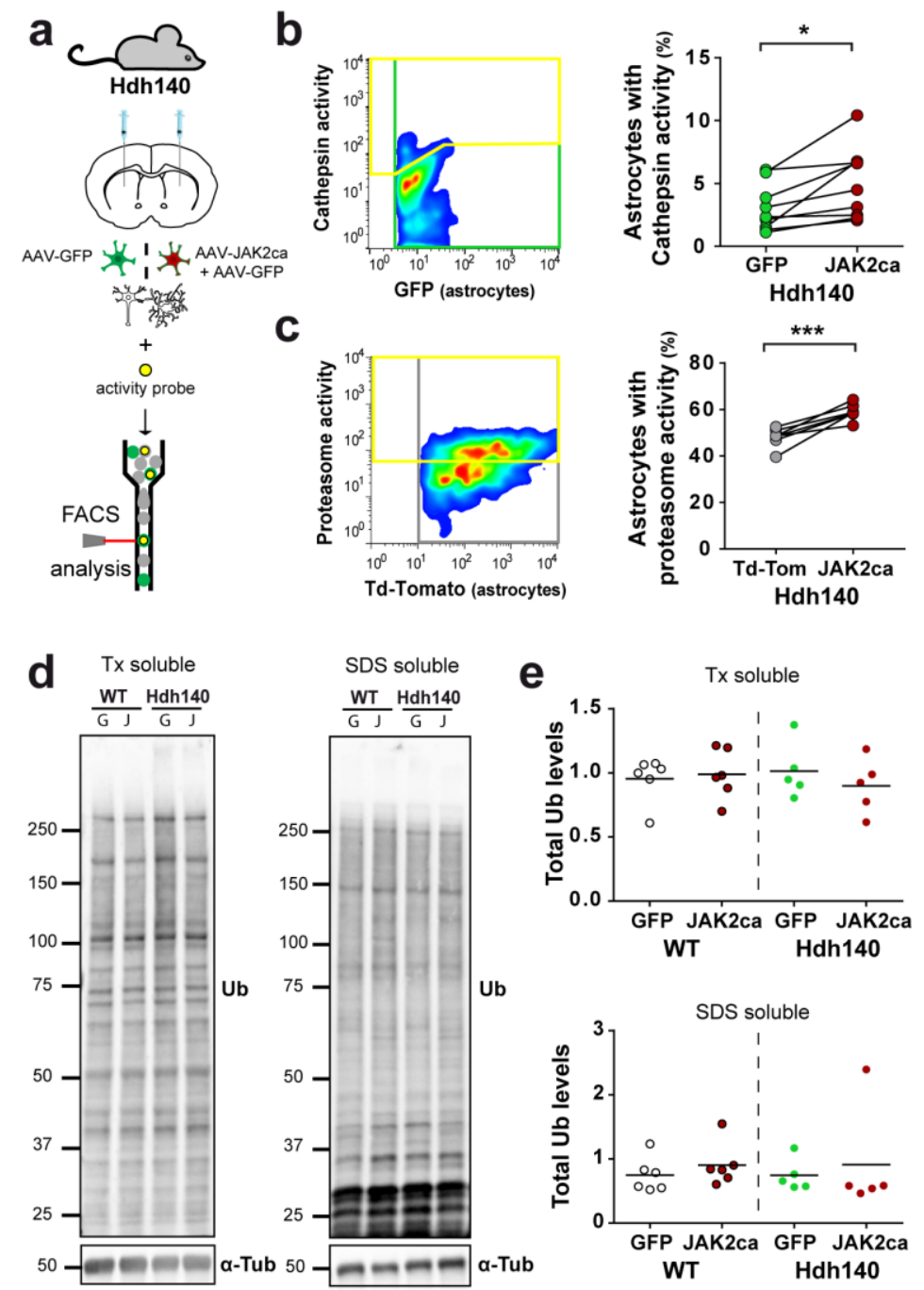

Figure 5. JAK2ca increases cathepsin and proteasome activities in HD striatal astrocytes

a-c. The striatum of Hdh-JAK2ca mice and their control Hdh140-GFP or Hdh140-Td-Tomato was collected, cells dissociated and incubated with a quenched fluorescent pan-cathepsin activity probe (b) or a proteasome activity probe (c). The percentage of $\mathrm{GFP}^{+}$astrocytes with cathepsin activity (b) or the percentage of $\mathrm{Td}^{-T_{0}}$ ato $^{+}$astrocytes with proteasome activity (c) was quantified in each mouse.

797 Hdh140-JAK2ca mice display a higher percentage of astrocytes with cathepsin or proteasome activity.

798 d, e. Immunoblotting for Ub was performed on striatal Tx-soluble and SDS-soluble fractions from WT799 GFP, WT-JAK2ca, Hdh140-GFP and Hdh140-JAK2ca mice. Immunoreactivity pattern and total Ub 800 levels are not different between groups. Band intensity was normalized to $\alpha$-tubulin $(\alpha$-tub). b, c. Paired $t$-test on arcsine-transformed data. $\mathbf{b} . \mathrm{N}=9$ /group. $\mathbf{c} . \mathrm{N}=7$ /group. d. ANOVA. $\mathrm{N}=5$-6/group. 

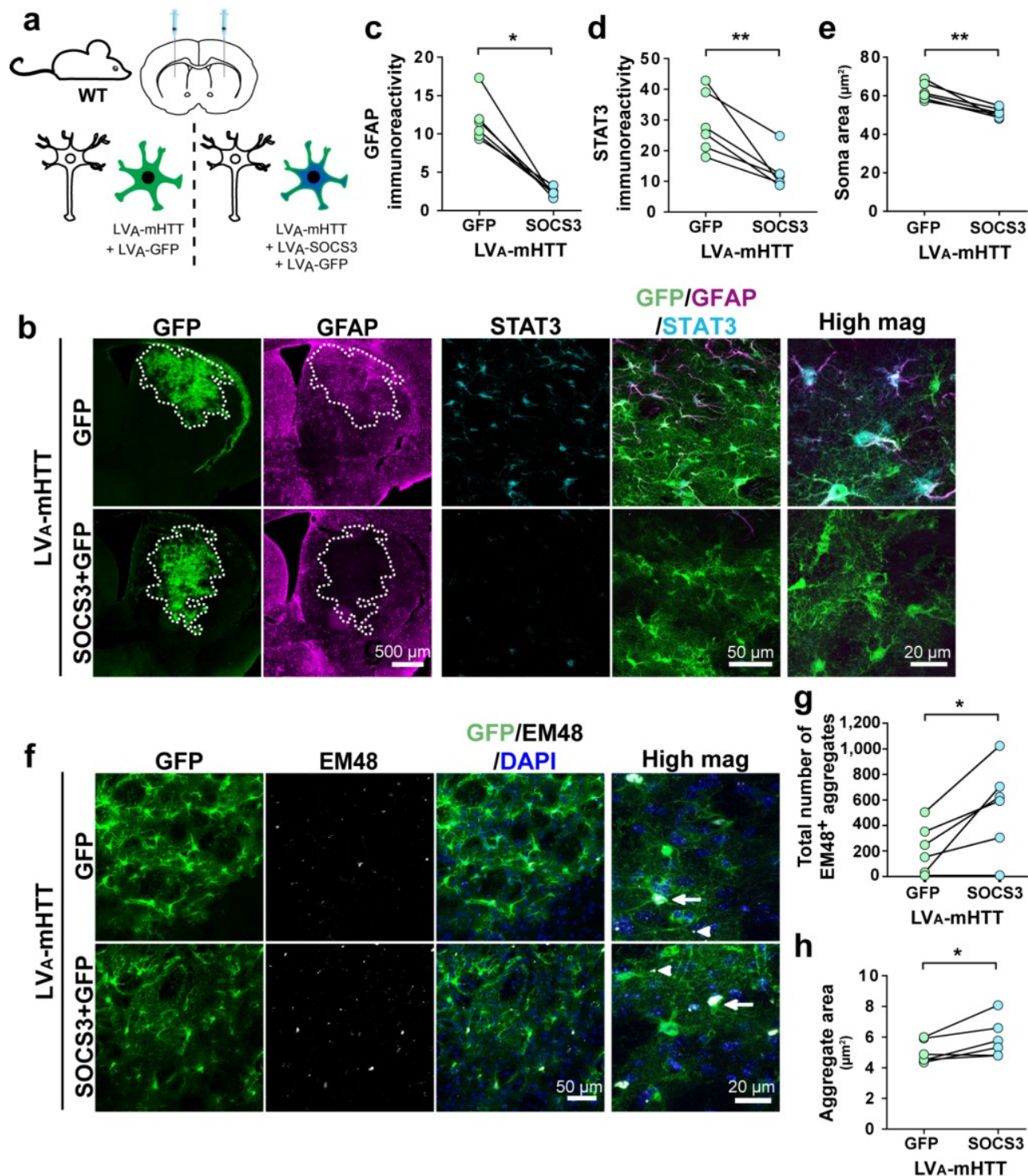

\section{HD astrocytes}

804 a. Two month-old WT mice were injected in one striatum with $L V_{\mathrm{A}}-\mathrm{mHTT}$ and $\mathrm{LV}_{\mathrm{A}}-\mathrm{GFP}$ and the contralateral striatum with $\mathrm{LV}_{\mathrm{A}}-\mathrm{mHTT}, \mathrm{LV}_{\mathrm{A}}-\mathrm{SOCS} 3$ and $\mathrm{LV}_{\mathrm{A}}-\mathrm{GFP}$ (same total viral load) to force mHTT expression in astrocytes. Their brains were analysed 6 weeks later. b. Low magnification images showing $\mathrm{GFP}^{+}$transduced area (outlined, green) and GFAP staining (magenta) in the mouse striatum. Confocal images of striatal sections stained for GFP (green), GFAP (magenta) and STAT3 (cyan). SOCS3 reduces GFAP immunoreactivity and nuclear accumulation of STAT3 in astrocytes. c-e. Quantification of GFAP immunoreactivity (c), STAT3 immunoreactivity (d) and astrocyte soma area (e). SOCS3 significantly decreases all these parameters. f. Confocal images of striatal sections stained

812 for GFP (green), EM48 (white) and DAPI (blue). Large EM48 ${ }^{+}$aggregates form in astrocyte nucleus 813 (arrow), while small aggregates are mainly found in astrocyte processes (arrowhead). $\mathbf{g}$, $\mathbf{h}$. The total 
bioRxiv preprint doi: https://doi.org/10.1101/2021.04.29.441924; this version posted April 29, 2021. The copyright holder for this preprint (which was not certified by peer review) is the author/funder, who has granted bioRxiv a license to display the preprint in perpetuity. It is made available under aCC-BY-NC-ND 4.0 International license.

814 number (g) and the size (h) of EM48 ${ }^{+}$aggregates are significantly increased by SOCS3. c. Wilcoxon 815 paired test. d, e, $\mathbf{g}, \mathbf{h}$. Paired $t$-test. $\mathrm{N}=6 /$ group. 


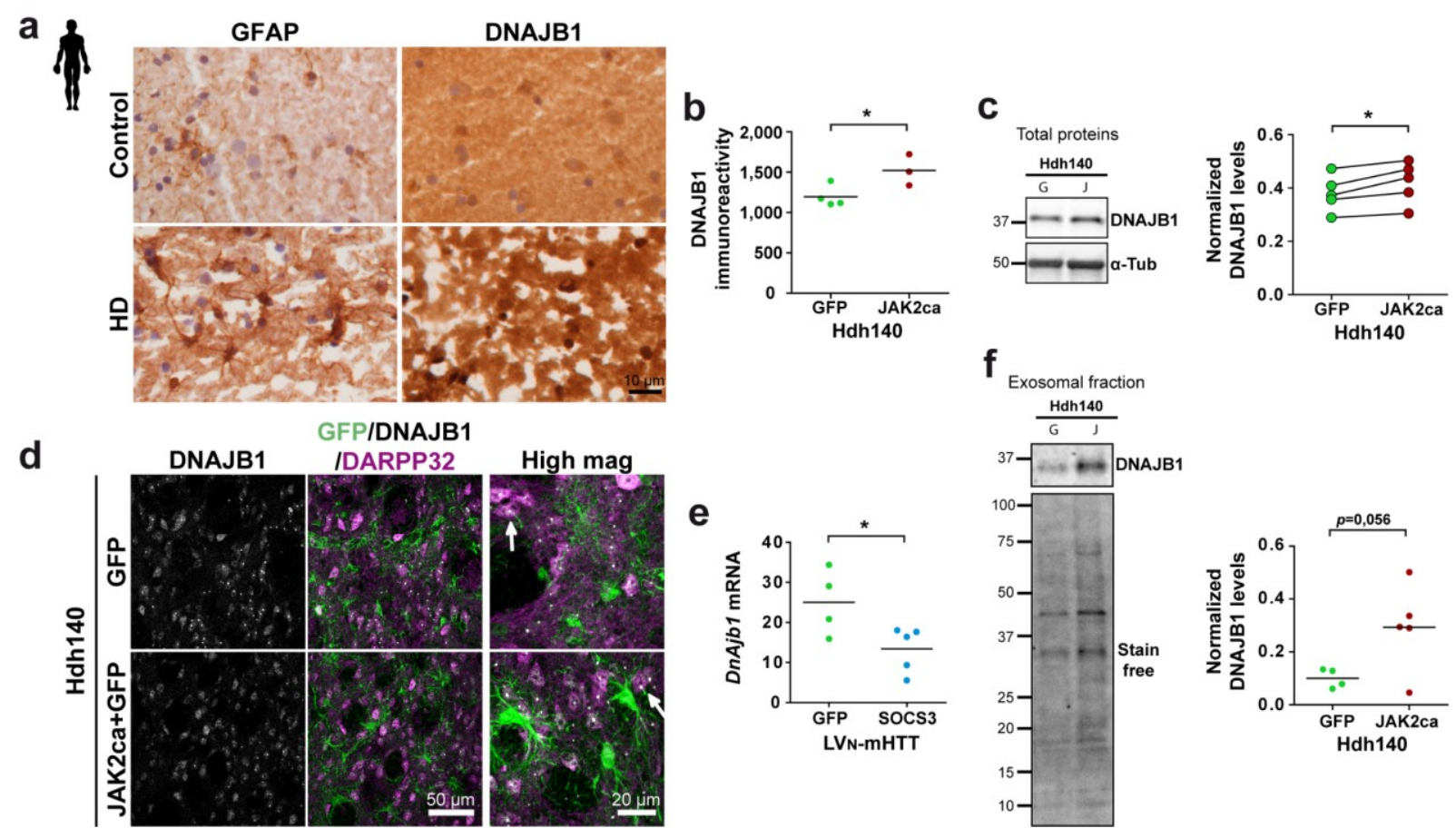

\section{Figure 7. The JAK2-STAT3 pathway increases DNAJB1 expression in astrocytes}

817 a. DNAJB1 display a strong immunoreactivity in the degenerative putamen region that is filled with

$818 \mathrm{GFAP}^{+}$astrocytes in HD patients. b, c. Immunostaining (b) and immunoblotting (c) show higher 819 DNAJB1 protein levels in the striatum of Hdh140-JAK2ca mice than Hdh140-GFP mice. Band intensity 820 was normalized to $\alpha$-tubulin ( $\alpha$-tub). d. Confocal images of striatal sections stained for GFP (green), 821 DNAJB1 (white) and DARPP32 (magenta). DNAJB1 displays a diffuse cytosolic staining and forms 822 small nuclear inclusions in neurons (arrow) of Hdh140 mice. e. Dnajb1 mRNA levels are significantly 823 decreased by SOCS3 in LV $\mathrm{N}_{\mathrm{N}}-\mathrm{mHTT}$ mice. f. DNAJB1 is present in exosomes isolated from Hdh140 824 striata and its levels, normalized by stain free staining, tend to be higher in Hdh140-JAK2ca than 825 Hdh140-GFP exosomes. b, e, f. Student $t$-test. N = 3-5/group. c. Paired $t$-test. N = 5/group. 

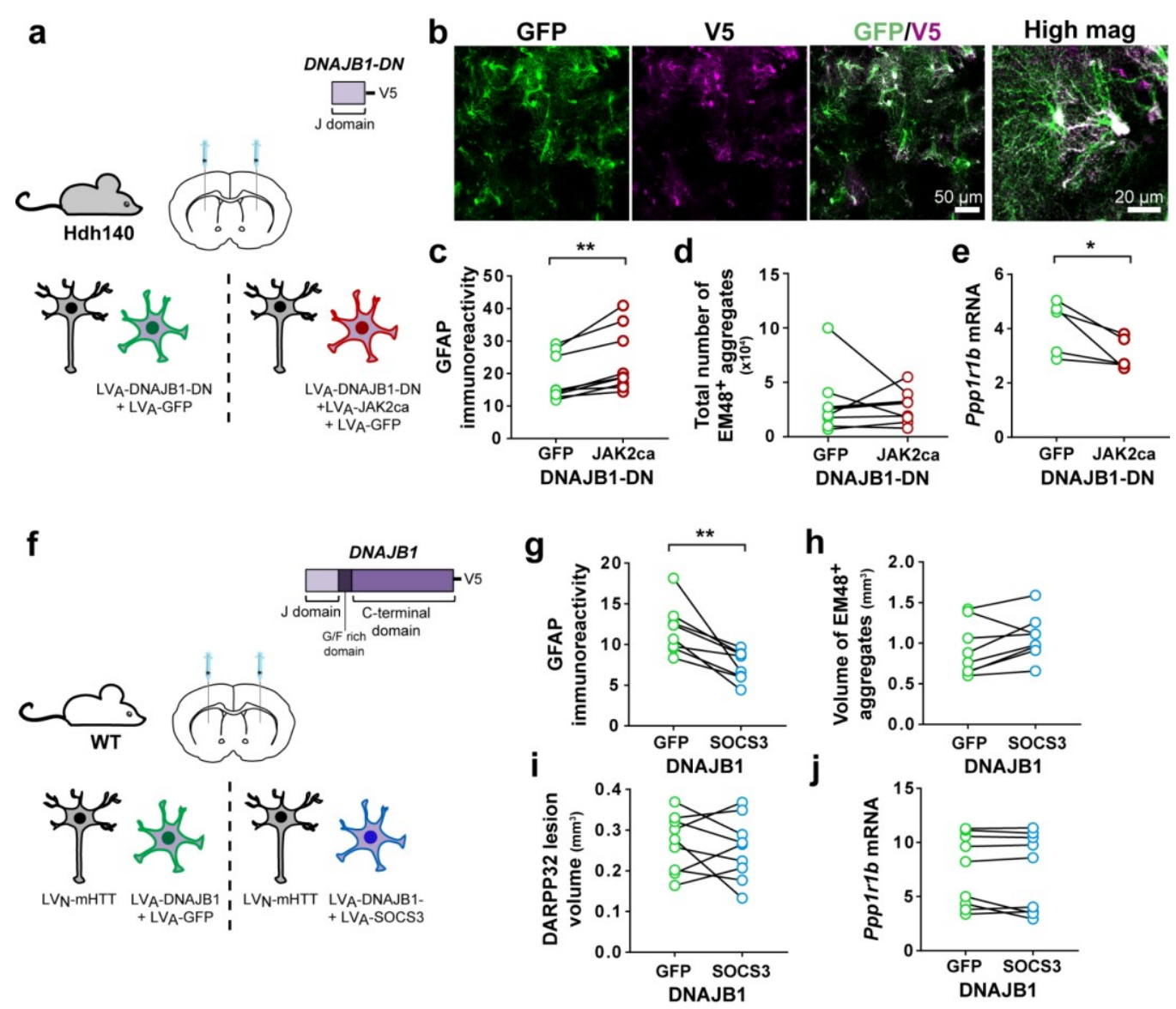

Figure 8. DNAJB1 is involved in the anti-aggregation effects of the JAK2-STAT3

\section{7}

828

829

830

831

832

833

834

835

836

837

838

839

840

841

\section{pathway}

a. Hdh140 mice (8-9 month-old) were injected in one striatum with $\mathrm{LV}_{\mathrm{A}}-\mathrm{GFP}+\mathrm{LV}_{\mathrm{A}}-\mathrm{DNAJB} 1-\mathrm{DN}$ and with $\mathrm{LV}_{\mathrm{A}}-\mathrm{JAK} 2 \mathrm{ca}+\mathrm{LV}_{\mathrm{A}}-\mathrm{GFP}+\mathrm{LV}_{\mathrm{A}}-\mathrm{DNAJB} 1-\mathrm{DN}$ in the contralateral striatum, at the same total virus load. Their brains were analysed 4 months later. b. Representative confocal images showing striatal sections stained for GFP (green) and V5 (magenta) in astrocytes. c. JAK2ca increases GFAP immunoreactivity in astrocytes overexpressing DNAJB1-DN in Hdh140 mice. d. In presence of DNAJB1-DN, the total number of EM48 ${ }^{+}$aggregates is no longer decreased by JAK2ca. e. Ppp1r1b mRNA levels are significantly reduced by JAK2ca in Hdh140 mice expressing DNAJB1-DN. f. Two month-old WT mice were injected in one striatum with $\mathrm{LV}_{\mathrm{N}}-\mathrm{mHTT}+\mathrm{LV}_{\mathrm{A}}-\mathrm{GFP}+\mathrm{LV}_{\mathrm{A}}$-DNAJB1 and the contralateral striatum with $\mathrm{LV}_{\mathrm{N}}-\mathrm{mHTT}+\mathrm{LV}_{\mathrm{A}}-\mathrm{SOCS} 3+\mathrm{LV}_{\mathrm{A}}-\mathrm{DNAJB} 1$, at the same total virus load

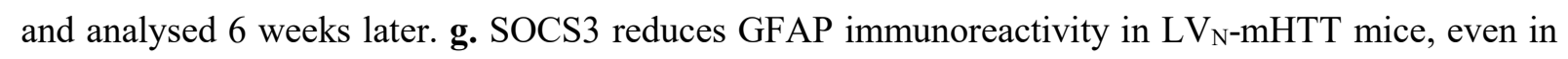
presence of DNJAB1. h. SOCS3 no longer increases the EM48 ${ }^{+}$volume when astrocytes co-express DNAJB1. i, j. Likewise, striatal DARPP32- lesions (i) and Ppp1r1b mRNA levels (j) are no longer different between the two groups. c-e, j. Wilcoxon paired test. $\mathrm{N}=8 /$ group (c, d). $\mathrm{N}=5 /$ group (e). g-h. Paired $t$-test. $\mathrm{N}=9$ /group. 


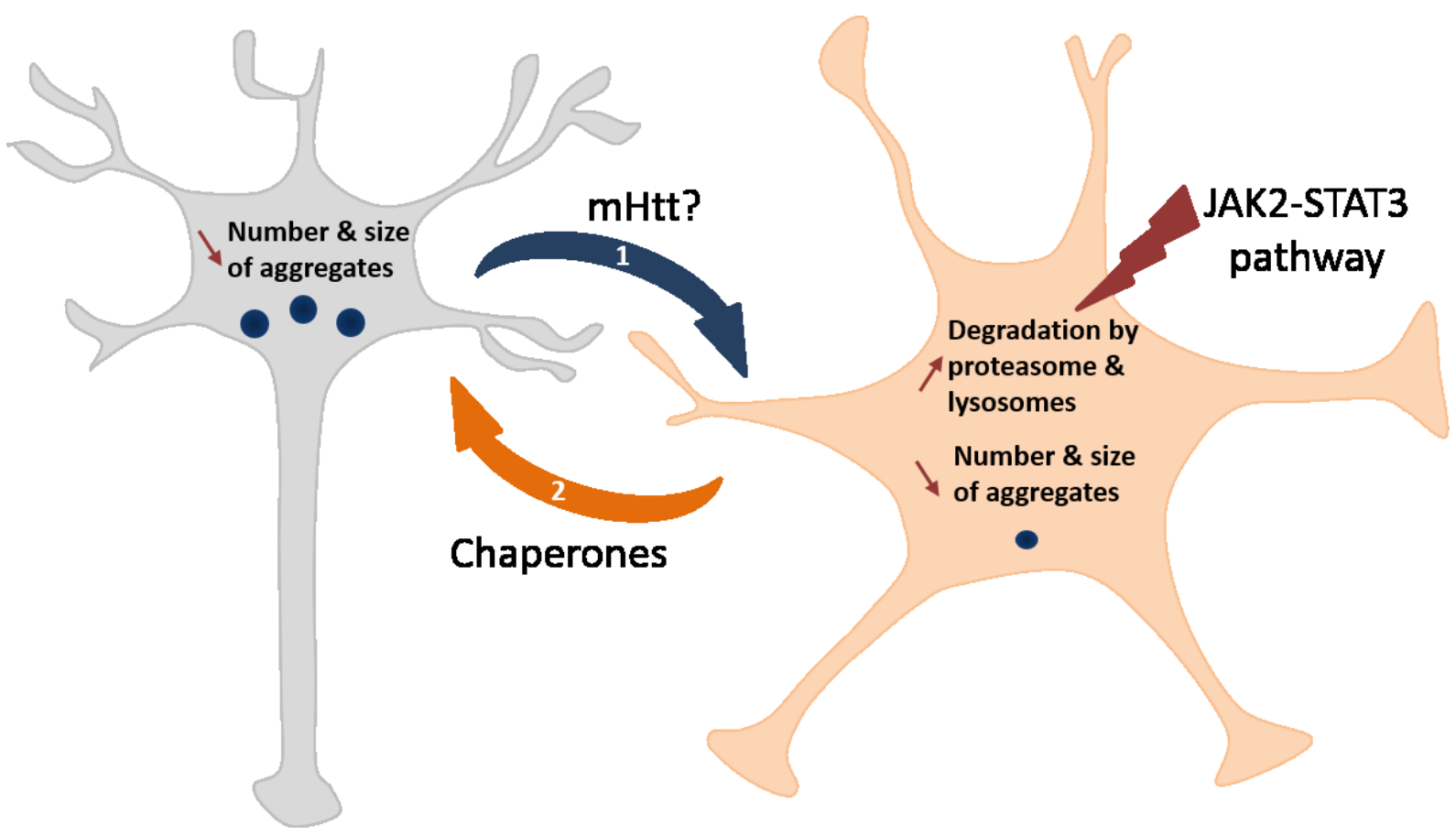

842 Figure 9. Working model: A bi-directional communication between JAK2-

843 STAT3-induced reactive astrocytes and neurons to promote proteostasis in HD

844 The JAK2-STAT3 pathway induces a reactive response in striatal astrocytes and activates a

845 transcriptional program that promotes proteostasis, reduces mHTT aggregation and improves neuronal

846 status. Two complementary and non-exclusive mechanisms involving striatal neurons and JAK2-

847 STAT3-dependent reactive astrocytes may take place. 1. Reactive astrocytes display a higher intrinsic

848 proteolytic activity that promotes mHTT degradation. Neurons could derive their mHTT to astrocytes

849 for clearance. The exact mechanisms and the form of mHTT exchanged (e.g. monomers, fibrils, cleaved

850 fragments) remain to be determined. 2. Reactive astrocytes over-express chaperones such as DNAJB1

851 that can be released in exosomes and promote proteostasis within neurons. 
bioRxiv preprint doi: https://doi.org/10.1101/2021.04 29.441924; this version posted April 29, 2021. The copyright holder for this preprint (which was not certified by peer review) is the author/funder, who has granted bioRxiv a license to display the preprint in perpetuity. It is made available under aCC-BY-NC-ND 4.0 International license.

852 Table 1. STAT3 involvement in gene regulation in HD astrocytes.

\begin{tabular}{|c|c|c|}
\hline Tool & $\begin{array}{c}\text { STAT3 rank among all significant } \\
\text { transcription factors }\end{array}$ & $\begin{array}{c}\boldsymbol{p} \text { value } \\
\text { (provided by each tool) }\end{array}$ \\
\hline ChEA3-Enrichr & $53 / 1122$ & $1.69 \mathrm{E}-22$ \\
\hline ChEA3-ENCODE & $96 / 104$ & $2.11 \mathrm{E}-2$ \\
\hline ChEA3-ReMap Atlas & $11 / 123$ & $1.91 \mathrm{E}-5$ \\
\hline HOMER & $25 / 55$ & $1.00 \mathrm{E}-9$ \\
\hline TRANSFAC & $46 / 75$ & $2.53 \mathrm{E}-6$ \\
\hline
\end{tabular}

853 STAT3 is identified among potential upstream transcription factors that regulate gene expression in

854 HD astrocytes. Data from Al-Dalahmah et al. $\stackrel{5}{\text {. }}$ 
a

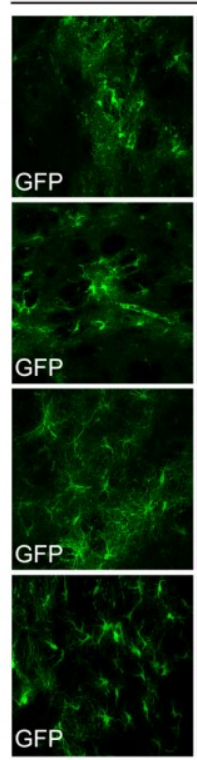

LV
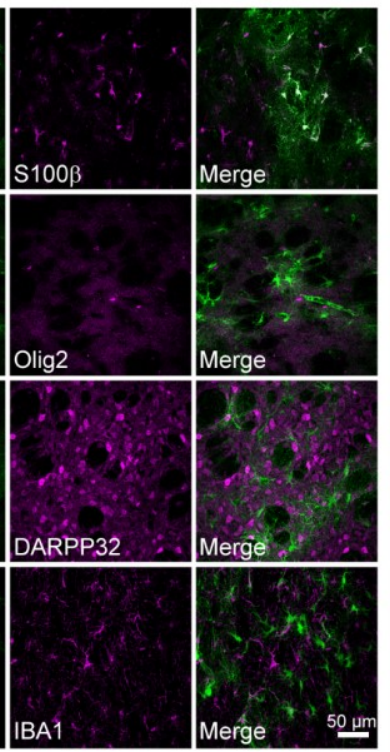

b Cell selection
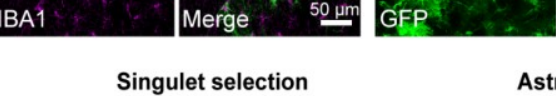

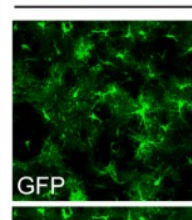

AAV

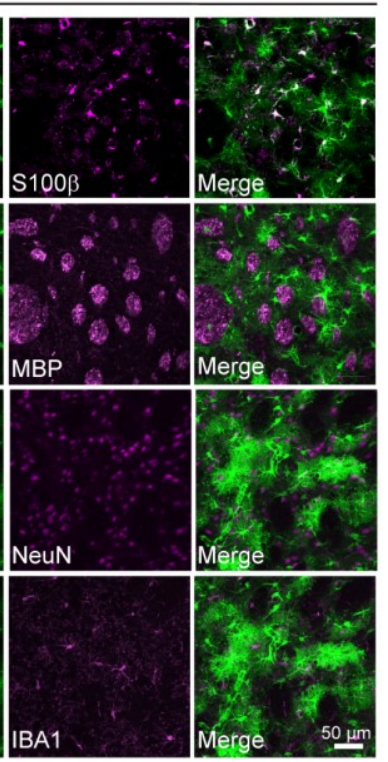

C
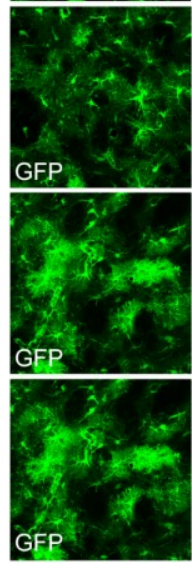

Astrocyte selection
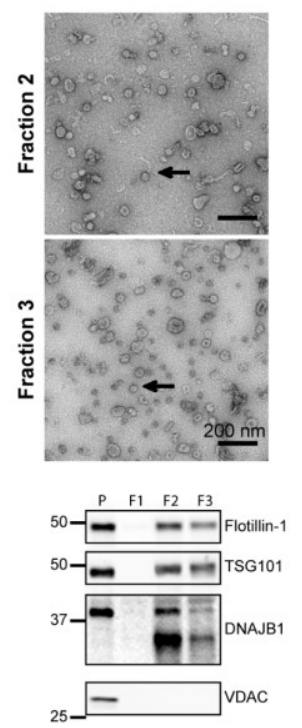

Cathepsin activity in astrocytes

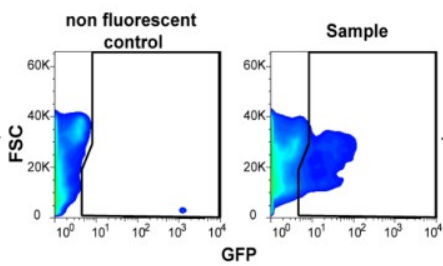

GFP

Astrocyte selection

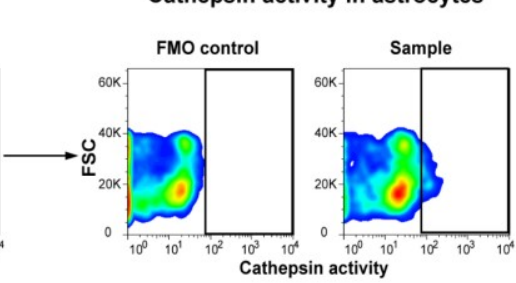

Cathepsin activity

Cell selection

Singulet selection non fluorescent
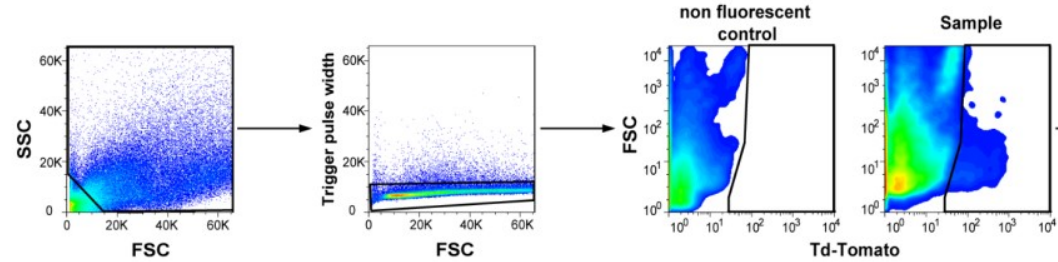

Proteasome activity in astrocytes

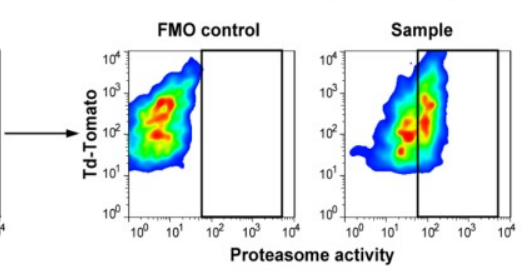

855

\section{Supplemental figure 1. Validation of experimental procedures}

a. Validation of astrocyte transduction by viral vectors. After injection of LV or AAV encoding GFP and targeting astrocytes in the mouse striatum, $\mathrm{GFP}^{+}$cells (green) co-express the astrocytic markers S100ß, but not IBA1, NeuN, DARPP32, MBP or Olig2 (magenta), which are specific markers for microglia, neurons, oligodendrocyte or cells of the oligodendrocyte lineage respectively, demonstrating selective astrocyte tropism of both vectors. b. Gating strategy for cathepsin and proteasome activity measurement on dissociated cells. Cells were gated on a side scatter/forward scatter plot, singlets were selected and then $\mathrm{GFP}^{+}$or Td-Tomato ${ }^{+}$astrocytes were gated based on a control non-fluorescent sample.

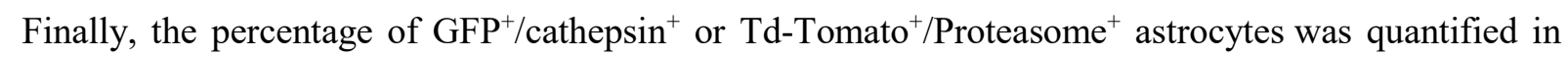
each mouse after setting the gates on a sample processed similarly without fluorescent activity probe (FMO control). c. Purity of the exosomal fractions. TEM of fractions 2 and 3 (F2, F3) obtained from the striatum of control mice evidences many circular vesicles with the typical size and shape of exosomes (arrow). Immunoblotting confirms that Flotillin-1 and TSG101, two exosomal proteins are 
bioRxiv preprint doi: https://doi.org/10.1101/2021.04.29.441924; this version posted April 29, 2021. The copyright holder for this preprint (which was not certified by peer review) is the author/funder, who has granted bioRxiv a license to display the preprint in perpetuity. It is made available under aCC-BY-NC-ND 4.0 International license.

enriched in F2 and F3, while VDAC, a mitochondrial protein, is only found in the first pellet (P) of total/brain homogenate. DNAJB1 is abundant in exosomal fractions. 


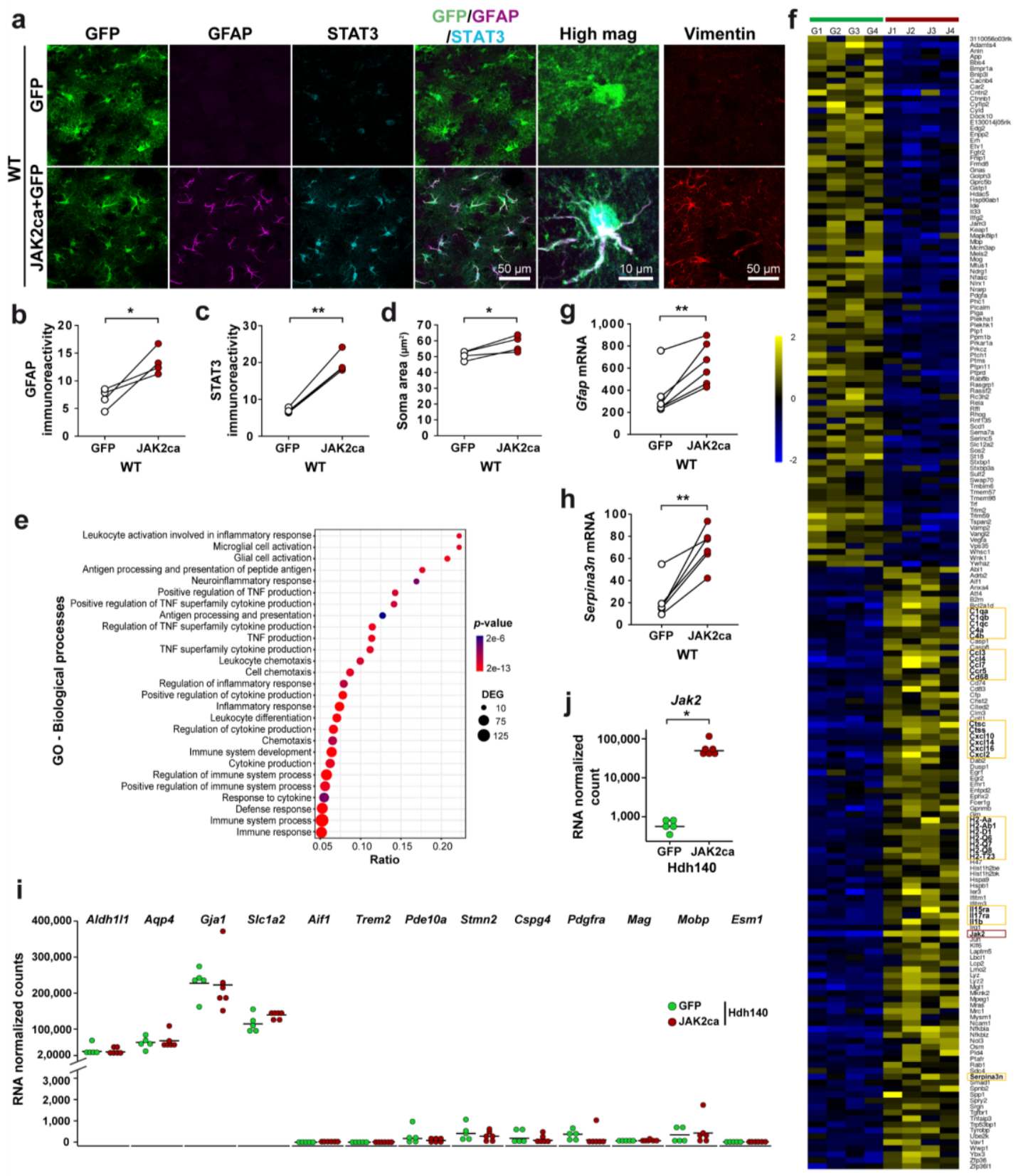

870 Supplemental figure 2. JAK2ca induces reactive changes in striatal astrocytes.

871 a-h. Mice were injected as in Fig. 4a. a-d. Representative confocal images showing brain sections

872 stained for GFP (green), GFAP (magenta), STAT3 (cyan) and vimentin (red) in WT-GFP and WT-

873 JAK2ca mice (a). JAK2ca increases GFAP and vimentin immunoreactivity, triggers STAT3

874 accumulation in the nucleus and induces soma hypertrophy in astrocytes (quantification in $\mathbf{b}, \mathbf{c}, \mathbf{d}$ ). e, $\mathbf{f}$.

875 Microarray analysis on FACS-sorted GFP-astrocytes $(\mathrm{G})$ and JAK2ca-astrocytes (J). GO analysis

876 identifies several biological processes linked to immunity and inflammation. DEG $=$ differentially

877 expressed genes (e). JAK2ca induces the expression of many genes involved in these biological

878 processes (f). Color scale represents mean-centered expression (log2-transformed). Cytokines and

879 chemokines, antigen presentation molecules and complement factors are highlighted in yellow boxes.

880 Note that Jak2 is also found upregulated (red box). g, h. JAK2ca-induction of Gfap (g) and Serpina3n 
881 (h) mRNA is validated by qPCR on bulk striatal samples prepared from GFP- and JAK2ca-mice. $\mathbf{i}, \mathbf{j}$.

882 Mice were injected as in Fig. 4d. Sorted astrocytes from both Hdh140-GFP and Hdh140-JAK2ca mice

883 express high levels of several astrocyte specific genes, while several markers of other cell types are

884 barely detectable. j. Jak2 mRNA are increased 100 fold in JAK2ca-astrocytes. b-d, f-h. Paired $t$-test. b-

885 d. $\mathrm{N}=4$ /group. g, h. $\mathrm{N}=6$ /group. $\mathbf{i}$-j. $\mathrm{N}=5$-6/group. 
Supplemental table 1: Sequences of RT-qPCR primers.

\begin{tabular}{|c|c|c|}
\hline Gene & Forward primer & Reverse primer \\
\hline Dnajb1 & CCTACGACGTGCTCAGCGAC & ATGGGGGTCTCCGTGGAAT \\
\hline Eef1 & CTACCCTCCACTTGGTCGCTT & GCAACTGTCTGCCTCATGTCAC \\
\hline Erp29 & CCTTCCCTTGGACACAGTCACT & GTCGAACTTCACCAAGACGAACTT \\
\hline Gfap & ACGACTATCGCCGCCAACT & GCCGCTCTAGGGACTCGTTC \\
\hline Htt & GCCGCCTCCGCCTCAAC & ATAGCGATGCCCAAGAGTTTC \\
\hline mHTT & GCCGCCTCCTCAGCTTC & ATAGCGATGCCCAAGAGTTTC \\
\hline Ppia & ATGGCAAATGCTGGACCAAA & GCСТTCTTTCACCTTCCCAAA \\
\hline Ppp1r1b & TCGGAGGAAGAGGATGAGTT & GGGGCTGGGATGCTGAGGTT \\
\hline Rpl13a & CTGAAGCCTACCAGAAAGTTTGC & GGTACTTCCACCCGACCTCAT \\
\hline Serpina3n & CAACCTTACAGGCCAACCCAT & GGGCACCAAGTAGTCCTAGATGCT \\
\hline Vimentin & TCGAGGTGGAGCGGGACAAC & TGCAGGGTGCTTTCGGCTTC \\
\hline
\end{tabular}

Supplemental table 2: Information on human samples.

\begin{tabular}{|c|c|c|c|c|c|}
\hline Group & Cause of death & Sex & Age (y) & $\begin{array}{c}\text { Post mortem } \\
\text { delay (h:min) }\end{array}$ & $\begin{array}{c}\text { Brain weight } \\
\text { (g) }\end{array}$ \\
\hline \multirow{4}{*}{$\begin{array}{c}\text { Non-demented } \\
\text { Control }\end{array}$} & $\begin{array}{c}\text { Mediastinum } \\
\text { carcinoma }\end{array}$ & Male & 45 & $08: 50$ & 1440 \\
\cline { 2 - 6 } & Euthanasia (Pain) & Female & 55 & $07: 30$ & 1260 \\
\cline { 2 - 6 } & Euthanasia (Pain) & Female & 60 & $05: 30$ & 1215 \\
\cline { 2 - 6 } & $\begin{array}{c}\text { Myocardial } \\
\text { infarction }\end{array}$ & Male & 73 & $09: 10$ & 1500 \\
\hline \multirow{4}{*}{ HD } & Euthanasia & Female & 38 & $05: 40$ & 1140 \\
\cline { 2 - 6 } & Euthanasia & Female & 57 & $06: 40$ & 1280 \\
\cline { 2 - 6 } & $\begin{array}{c}\text { Respiratory } \\
\text { insufficiency }\end{array}$ & Female & 64 & $05: 00$ & 975 \\
\cline { 2 - 6 } & Euthanasia & Male & 71 & $04: 25$ & 1320 \\
\hline
\end{tabular}


bioRxiv preprint doi: https://doi.org/10.1101/2021.04.29.441924; this version posted April 29, 2021. The copyright holder for this preprint (which was not certified by peer review) is the author/funder, who has granted bioRxiv a license to display the preprint in perpetuity. It is made available under aCC-BY-NC-ND 4.0 International license.

\section{References}

1. Tabrizi, S.J., Flower, M.D., Ross, C.A. \& Wild, E.J. Huntington disease: new insights into molecular pathogenesis and therapeutic opportunities. Nature reviews. Neurology 16, 529-546 (2020).

2. The Huntington study group. A novel gene containing a trinucleotide repeat that is expanded and unstable on Huntington's disease chromosomes. The Huntington's Disease Collaborative Research Group. Cell 72, 971-983 (1993).

3. DiFiglia, M., et al. Aggregation of huntingtin in neuronal intranuclear inclusions and dystrophic neurites in brain. Science 277, 1990-1993 (1997).

4. Jansen, A.H., et al. Frequency of nuclear mutant huntingtin inclusion formation in neurons and glia is cell-type-specific. Glia 65, 50-61 (2017).

5. Al-Dalahmah, O., et al. Single-nucleus RNA-seq identifies Huntington disease astrocyte states. Acta neuropathologica communications 8, 19 (2020).

6. Vonsattel, J.P., et al. Neuropathological classification of Huntington's disease. Journal of neuropathology and experimental neurology 44, 559-577 (1985).

7. Selkoe, D.J., Salazar, F.J., Abraham, C. \& Kosik, K.S. Huntington's disease: changes in striatal proteins reflect astrocytic gliosis. Brain Res 245, 117-125 (1982).

8. Faideau, M., et al. In vivo expression of polyglutamine-expanded huntingtin by mouse striatal astrocytes impairs glutamate transport: a correlation with Huntington's disease subjects. Hum Mol Genet 19, 3053-3067 (2010).

9. Lee, H., et al. Cell Type-Specific Transcriptomics Reveals that Mutant Huntingtin Leads to Mitochondrial RNA Release and Neuronal Innate Immune Activation. Neuron 107, 891-908 e898 (2020).

10. Verkhratsky, A. \& Nedergaard, M. Physiology of Astroglia. Physiological reviews 98, 239-389 (2018).

11. Khakh, B.S., et al. Unravelling and Exploiting Astrocyte Dysfunction in Huntington's Disease. Trends Neurosci 40, 422-437 (2017).

12. Lievens, J.C., et al. Impaired glutamate uptake in the R6 Huntington's disease transgenic mice. Neurobiol Dis 8, 807-821 (2001).

13. Tong, X., et al. Astrocyte Kir4.1 ion channel deficits contribute to neuronal dysfunction in Huntington's disease model mice. Nat Neurosci 17, 694-703 (2014).

14. Hsiao, H.Y., et al. Aberrant astrocytes impair vascular reactivity in Huntington disease. Ann Neurol 78, 178-192 (2015).

15. Rebec, G.V., Barton, S.J. \& Ennis, M.D. Dysregulation of ascorbate release in the striatum of behaving mice expressing the Huntington's disease gene. J Neurosci 22, RC202 (2002).

16. Hong, Y., Zhao, T., Li, X.J. \& Li, S. Mutant Huntingtin Impairs BDNF Release from Astrocytes by Disrupting Conversion of Rab3a-GTP into Rab3a-GDP. J Neurosci 36, 8790-8801 (2016).

17. Wojtowicz, A.M., Dvorzhak, A., Semtner, M. \& Grantyn, R. Reduced tonic inhibition in striatal output neurons from Huntington mice due to loss of astrocytic GABA release through GAT-3. Frontiers in neural circuits 7, 188 (2013).

18. Hong, Y., Zhao, T., Li, X.J. \& Li, S. Mutant Huntingtin Inhibits alphaB-Crystallin Expression and Impairs Exosome Secretion from Astrocytes. J Neurosci 37, 9550-9563 (2017).

19. Yu, X., et al. Context-Specific Striatal Astrocyte Molecular Responses Are Phenotypically Exploitable. Neuron (2020).

20. Diaz-Castro, B., Gangwani, M.R., Yu, X., Coppola, G. \& Khakh, B.S. Astrocyte molecular signatures in Huntington's disease. Science translational medicine 11 (2019).

21. Ceyzériat, K., Abjean, L., Carrillo-de Sauvage, M.A., Ben Haim, L. \& Escartin, C. The complex STATes of astrocyte reactivity: How are they controlled by the JAK-STAT3 pathway? Neuroscience 330, 205-218 (2016).

22. Ben Haim, L., et al. The JAK/STAT3 pathway is a common inducer of astrocyte reactivity in Alzheimer's and Huntington's diseases. J Neurosci 35, 2817-2829 (2015).

23. Schaffar, G., et al. Cellular toxicity of polyglutamine expansion proteins: mechanism of transcription factor deactivation. Molecular cell 15, 95-105 (2004). 
bioRxiv preprint doi: https://doi.org/10.1101/2021.04.29.441924; this version posted April 29, 2021. The copyright holder for this preprint (which was not certified by peer review) is the author/funder, who has granted bioRxiv a license to display the preprint in perpetuity. It is made available under aCC-BY-NC-ND 4.0 International license.

24. Hosp, F., et al. Spatiotemporal Proteomic Profiling of Huntington's Disease Inclusions Reveals Widespread Loss of Protein Function. Cell reports 21, 2291-2303 (2017).

25. Arrasate, M., Mitra, S., Schweitzer, E.S., Segal, M.R. \& Finkbeiner, S. Inclusion body formation reduces levels of mutant huntingtin and the risk of neuronal death. Nature 431, 805-810 (2004).

26. Ortega, Z. \& Lucas, J.J. Ubiquitin-proteasome system involvement in Huntington's disease. Frontiers in molecular neuroscience 7, 77 (2014).

27. Cortes, C.J. \& La Spada, A.R. The many faces of autophagy dysfunction in Huntington's disease: from mechanism to therapy. Drug discovery today 19, 963-971 (2014).

28. Tydlacka, S., Wang, C.E., Wang, X., Li, S. \& Li, X.J. Differential activities of the ubiquitinproteasome system in neurons versus glia may account for the preferential accumulation of misfolded proteins in neurons. J Neurosci 28, 13285-13295 (2008).

29. Zhao, T., Hong, Y., Yin, P., Li, S. \& Li, X.J. Differential HspBP1 expression accounts for the greater vulnerability of neurons than astrocytes to misfolded proteins. Proc Natl Acad Sci U S A 114, E7803-E7811 (2017).

30. Jansen, A.H., Reits, E.A. \& Hol, E.M. The ubiquitin proteasome system in glia and its role in neurodegenerative diseases. Frontiers in molecular neuroscience 7, 73 (2014).

31. Sung, K. \& Jimenez-Sanchez, M. Autophagy in Astrocytes and its Implications in Neurodegeneration. Journal of molecular biology 432, 2605-2621 (2020).

32. Koyuncu, S., Fatima, A., Gutierrez-Garcia, R. \& Vilchez, D. Proteostasis of Huntingtin in Health and Disease. International journal of molecular sciences 18 (2017).

33. Ciechanover, A. \& Kwon, Y.T. Protein Quality Control by Molecular Chaperones in Neurodegeneration. Frontiers in neuroscience 11, 185 (2017).

34. San Gil, R., Ooi, L., Yerbury, J.J. \& Ecroyd, H. The heat shock response in neurons and astroglia and its role in neurodegenerative diseases. Molecular neurodegeneration 12, 65 (2017).

35. Menalled, L.B., Sison, J.D., Dragatsis, I., Zeitlin, S. \& Chesselet, M.F. Time course of early motor and neuropathological anomalies in a knock-in mouse model of Huntington's disease with 140 CAG repeats. J Comp Neurol 465, 11-26 (2003).

36. Colin, A., et al. Engineered lentiviral vector targeting astrocytes in vivo. Glia 57, 667-679 (2009).

37. Naldini, L., Blomer, U., Gage, F.H., Trono, D. \& Verma, I.M. Efficient transfer, integration, and sustained long-term expression of the transgene in adult rat brains injected with a lentiviral vector. Proc Natl Acad Sci U S A 93, 11382-11388 (1996).

38. Lee, Y., Messing, A., Su, M. \& Brenner, M. GFAP promoter elements required for region-specific and astrocyte-specific expression. Glia 56, 481-493 (2008).

39. Ceyzeriat, K., et al. Modulation of astrocyte reactivity improves functional deficits in mouse models of Alzheimer's disease. Acta neuropathologica communications 6, 104 (2018).

40. Guillemaud, O., et al. Complex roles for reactive astrocytes in the triple transgenic mouse model of Alzheimer disease. Neurobiol Aging 90, 135-146 (2020).

41. de Almeida, L.P., Ross, C.A., Zala, D., Aebischer, P. \& Deglon, N. Lentiviral-mediated delivery of mutant huntingtin in the striatum of rats induces a selective neuropathology modulated by polyglutamine repeat size, huntingtin expression levels, and protein length. $J$ Neurosci 22, 3473 3483 (2002).

42. Michels, A.A., Kanon, B., Bensaude, O. \& Kampinga, H.H. Heat shock protein (Hsp) 40 mutants inhibit Hsp70 in mammalian cells. J Biol Chem 274, 36757-36763 (1999).

43. Vella, L.J., et al. A rigorous method to enrich for exosomes from brain tissue. Journal of extracellular vesicles $\mathbf{6}, 1348885$ (2017).

44. Kabani, M. \& Melki, R. Sup35p in Its Soluble and Prion States Is Packaged inside Extracellular Vesicles. mBio 6 (2015).

45. Smyth, G.K. limma: Linear Models for Microarray Data. in Bioinformatics and Computational Biology Solutions Using R and Bioconductor. Statistics for Biology and Health (ed. G. R., C. V.J., H. W., I. R.A. \& D. S.) (Springer, New York, NY, 2005).

46. Andrews, S. FastQC: A Quality Control Tool for High Throughput Sequence Data. Available online at: http://www.bioinformatics.babraham.ac.uk/projects/fastgcl (2010).

47. Kim, D., Langmead, B. \& Salzberg, S.L. HISAT: a fast spliced aligner with low memory requirements. Nature methods 12, 357-360 (2015). 
bioRxiv preprint doi: https://doi.org/10.1101/2021.04.29.441924; this version posted April 29, 2021. The copyright holder for this preprint (which was not certified by peer review) is the author/funder, who has granted bioRxiv a license to display the preprint in perpetuity. It is made available under aCC-BY-NC-ND 4.0 International license.

48. Liao, Y., Smyth, G.K. \& Shi, W. featureCounts: an efficient general purpose program for assigning sequence reads to genomic features. Bioinformatics 30, 923-930 (2014).

49. Love, M.I., Huber, W. \& Anders, S. Moderated estimation of fold change and dispersion for RNA-seq data with DESeq2. Genome Biol 15, 550 (2014).

50. Subramanian, A., et al. Gene set enrichment analysis: a knowledge-based approach for interpreting genome-wide expression profiles. Proc Natl Acad Sci U S A 102, 15545-15550 (2005).

51. Heinz, S., et al. Simple combinations of lineage-determining transcription factors prime cisregulatory elements required for macrophage and B cell identities. Molecular cell 38, 576-589 (2010).

52. Zambelli, F., Pesole, G. \& Pavesi, G. Pscan: finding over-represented transcription factor binding site motifs in sequences from co-regulated or co-expressed genes. Nucleic Acids Res 37, W247252 (2009).

53. Keenan, A.B., et al. ChEA3: transcription factor enrichment analysis by orthogonal omics integration. Nucleic Acids Res 47, W212-W224 (2019).

54. Hickey, M.A., et al. Extensive early motor and non-motor behavioral deficits are followed by striatal neuronal loss in knock-in Huntington's disease mice. Neuroscience 157, 280-295 (2008).

55. Escartin, C., et al. Reactive astrocyte nomenclature, definitions, and future directions. Nat Neurosci 24, 312-325 (2021).

56. Withana, N.P., et al. Labeling of active proteases in fresh-frozen tissues by topical application of quenched activity-based probes. Nature protocols 11, 184-191 (2016).

57. Berkers, C.R., et al. Profiling proteasome activity in tissue with fluorescent probes. Mol Pharm 4, 739-748 (2007).

58. Hartl, F.U., Bracher, A. \& Hayer-Hartl, M. Molecular chaperones in protein folding and proteostasis. Nature 475, 324-332 (2011).

59. Takeuchi, T., et al. Intercellular chaperone transmission via exosomes contributes to maintenance of protein homeostasis at the organismal level. Proc Natl Acad Sci US A 112, E2497-2506 (2015).

60. Pearce, M.M., Spartz, E.J., Hong, W., Luo, L. \& Kopito, R.R. Prion-like transmission of neuronal huntingtin aggregates to phagocytic glia in the Drosophila brain. Nature communications 6, 6768 (2015).

61. Pecho-Vrieseling, E., et al. Transneuronal propagation of mutant huntingtin contributes to noncell autonomous pathology in neurons. Nat Neurosci 17, 1064-1072 (2014).

62. Babcock, D.T. \& Ganetzky, B. Transcellular spreading of huntingtin aggregates in the Drosophila brain. Proc Natl Acad Sci U S A 112, E5427-5433 (2015).

63. Donnelly, K.M., et al. Phagocytic glia are obligatory intermediates in transmission of mutant huntingtin aggregates across neuronal synapses. Elife 9 (2020).

64. Cicchetti, F., et al. Mutant huntingtin is present in neuronal grafts in Huntington disease patients. Ann Neurol 76, 31-42 (2014).

65. Diaz-Hidalgo, L., et al. Transglutaminase type 2-dependent selective recruitment of proteins into exosomes under stressful cellular conditions. Biochim Biophys Acta 1863, 2084-2092 (2016).

66. Jeon, I., et al. Human-to-mouse prion-like propagation of mutant huntingtin protein. Acta Neuropathol 132, 577-592 (2016).

67. Costanzo, M., et al. Transfer of polyglutamine aggregates in neuronal cells occurs in tunneling nanotubes. $J$ Cell Sci 126, 3678-3685 (2013).

68. Trajkovic, K., Jeong, H. \& Krainc, D. Mutant Huntingtin Is Secreted via a Late Endosomal/Lysosomal Unconventional Secretory Pathway. J Neurosci 37, 9000-9012 (2017).

69. Kampinga, H.H. \& Craig, E.A. The HSP70 chaperone machinery: J proteins as drivers of functional specificity. Nature reviews. Molecular cell biology 11, 579-592 (2010).

70. Scior, A., et al. Complete suppression of $\mathrm{Htt}$ fibrilization and disaggregation of $\mathrm{Htt}$ fibrils by a trimeric chaperone complex. EMBO J 37, 282-299 (2018).

71. Wentink, A.S., et al. Molecular dissection of amyloid disaggregation by human HSP70. Nature 587, 483-488 (2020).

72. Popiel, H.A., et al. Hsp40 gene therapy exerts therapeutic effects on polyglutamine disease mice via a non-cell autonomous mechanism. PLoS One 7, e51069 (2012). 
bioRxiv preprint doi: https://doi.org/10.1101/2021.04 29.441924; this version posted April 29, 2021. The copyright holder for this preprint (which was not certified by peer review) is the author/funder, who has granted bioRxiv a license to display the preprint in perpetuity. It is made available under aCC-BY-NC-ND 4.0 International license.

73. Warrick, J.M., et al. Suppression of polyglutamine-mediated neurodegeneration in Drosophila by the molecular chaperone HSP70. Nature genetics 23, 425-428 (1999).

74. Bason, M., et al. Astrocytic expression of the chaperone DNAJB6 results in non-cell autonomous protection in Huntington's disease. Neurobiol Dis 124, 108-117 (2019).

75. Arrasate, M. \& Finkbeiner, S. Protein aggregates in Huntington's disease. Exp Neurol 238, 1-11 (2012).

76. Ramdzan, Y.M., et al. Huntingtin Inclusions Trigger Cellular Quiescence, Deactivate Apoptosis, and Lead to Delayed Necrosis. Cell reports 19, 919-927 (2017).

77. Pircs, K., et al. Huntingtin Aggregation Impairs Autophagy, Leading to Argonaute-2 Accumulation and Global MicroRNA Dysregulation. Cell reports 24, 1397-1406 (2018). 